\title{
Four simplified gradient elasticity models for the simulation of dispersive wave propagation
}

\author{
Harm Askes ${ }^{1 *}$ Andrei V. Metrikine ${ }^{2}$, Aleksey V. Pichugin ${ }^{3}$ and Terry Bennett ${ }^{1}$ \\ ${ }^{1}$ Department of Civil and Structural Engineering, \\ University of Sheffield, Sheffield, U.K. \\ ${ }^{2}$ Faculty of Civil Engineering and Geosciences, \\ Delft University of Technology, Delft, Netherlands \\ ${ }^{3}$ Department of Mathematical Sciences, \\ Brunel University, Uxbridge, U.K.
}

\begin{abstract}
Gradient elasticity theories can be used to simulate dispersive wave propagation as it occurs in heterogeneous materials. Compared to the second-order partial differential equations of classicaly elasticity, in its most general format gradient elasticity also contains fourth-order spatial, temporal as well as mixed spatial-temporal derivatives. The inclusion of the various higher-order terms has been motivated through arguments of causality and asymptotic accuracy, but for numerical implementations it is also important that standard discretisation tools can be used for the interpolation in space and the integration in time. In this paper, we will formulate four different simplifications of the general gradient elasticity theory. We will study the dispersive properties of the models, their causality according to Einstein and their behaviour in simple initial/boundary value problems.
\end{abstract}

\section{Introduction}

Wave propagation through a heterogeneous material is normally dispersive, that is, each harmonic wave component travels with a different velocity. In most materials, waves with larger wave numbers travel slower than waves with smaller wave numbers. Indeed, such dispersive wave propagation has been observed experimentally in a range of materials and through a range of scales, as for instance phonon dispersion in Bismuth [1]. When dispersive wave propagation is simulated, the analyst needs to balance accuracy and efficiency of the model. The classical equations of elasticity are non-dispersive, thus describing the material as homogeneous using classical elasticity is not an option. A detailed modelling of every heterogeneity in detail is normally prohibitive, but instead so-called generalised continuum theories can be used as an alternative.

Generalised continuum theories describe the material as homogeneous yet they contain additional terms that capture the heterogeneities. An important class of generalised continuum theories are the so-called gradient elasticity theories, in which the classical stress-strain relations are extended with additional gradients. These theories build upon the theories of elasticity with microstructure of Mindlin [2] and Toupin [3]. Simplified formats have been popularised in the 1990s through the works of Aifantis, e.g. [4] suggested the following constitutive relation which was motivated for use in statics

$$
\sigma_{i j}=C_{i j k l}\left(\varepsilon_{k l}-\ell^{2} \varepsilon_{k l, m m}\right)
$$

where $\sigma$ and $\varepsilon$ are the Cauchy stress and the infinitesimal strain, respectively, $C$ contains the elastic moduli and the additional parameter $\ell$ is an internal length scale. The format of Equation (1) has been used successfully to

*corresponding author; University of Sheffield, Department of Civil and Structural Engineering, Sir Frederick Mappin Building, Mappin Street, Sheffield S1 3JD, email h.askes@sheffield.ac.uk 
dispose of strain singularities in crack tip analysis [5-8] and dislocation analysis [9-11]. Another class of gradient elasticity theories have been formulated whereby the response of a discrete material model is continualised in order to capture the dynamic behaviour of heterogeneous materials [12-15]. The commonly obtained format reads

$$
\sigma_{i j}=C_{i j k l}\left(\varepsilon_{k l}+\ell^{2} \varepsilon_{k l, m m}\right)
$$

where the only difference with Equation (1) concerns the sign of the higher-order term. The advantages of such approaches are that the the internal length scale $\ell$ is normally straightforwardly identified in terms of the geometry of the heterogeneity, e.g. particle spacing, and the dispersive properties of discrete material models are approximated with more accuracy compared with classical elasticity. Unfortunately, however, models according to Equation (2) are unstable [16-18]; while they possess some merit in predicting the dispersive properties of materials they should not be used in boundary value problems.

Gradient elasticity theories that can be used in statics (e.g. removal of strain singularities) as well as in dynamics (e.g. realistic prediction of dispersion) should combine the advantages of Equations (1) and (2). Gradient theories with higher-order inertia terms fulfill this requirement; a generic format that is an extension of Equation (1) reads [19-24]

$$
\sigma_{i j}=C_{i j k l}\left(\varepsilon_{k l}-\ell_{s}^{2} \varepsilon_{k l, m m}\right)+\rho \ell_{m}^{2} u_{i, j t t}
$$

where two length scales are included: $\ell_{s}$ accompanies the higher-order stiffness term and $\ell_{m}$ accompanies the higher-order inertia term. The double time derivative of the last term in Equation (3) denotes inertia and should not be confused with viscosity (which would have been indicated with an odd time derivative). In fact, Equation (3) follows the generic formulation of Mindlin, who proposed a simultaneous extension of the potential energy and the kinetic energy [2]. Note that the static counterpart of Equation (3) follows Equation (1), not Equation (2).

Although Equation (3) predicts a realistic dispersion of propagating waves, nevertheless further improvements of Equation (3) have been suggested recently, namely the inclusion of a fourth-order time derivative in the equations of motion. The case has been argued indepedently in two different ways, namely:

- Metrikine [25] demonstrated that the model according to Equation (3) is non-causal in the sense that energy can propagate faster than the speed of light (albeit with infinitesimal amplitude). To mitigate this, i.e. to retain causality of the formulation, a fourth-order time derivative is required alongside fourth-order spatial derivatives and mixed fourth-order derivatives (twice with respect to time, twice with respect to the spatial coordinates). It was furthermore shown that such a model can be derived by continualisation of a two-phase material.

- Pichugin et al. [26] compared various series expansions and explored their asymptotic equivalence in the construction of gradient elasticity theories. The various higher-order derivatives are asymptotically equivalent but upon truncation give different approximation errors when compared to the response of a discrete model. The inclusion of the fourth-order time derivative can be used to improve the asymptotic accuracy of the resulting theory.

Independently of the above arguments of causality and asymptotic accuracy, a number of studies have addressed the formulation of gradient elasticity theories that lend themselves to straightforward numerical implementation, e.g. with finite element methods and standard time integration techniques. The equilibrium equations according to Equation (1) are fourth-order and would thus require $\mathscr{C}^{1}$-continuity of the spatial interpolation. While some progress in $\mathscr{C}^{1}$-continuous implementations for gradient theories has been made using Hermitian finite elements [27] and meshless methods [28,29], another line of research has been to re-formulate gradient elasticity prior to discretisation such that the usual $\mathscr{C}^{0}$-continuous shape functions can be used. Ru and Aifantis have suggested an operator split for gradient elasticity by which two second-order equations are solved sequentially, rather than the original fourth-order equations [6]. Numerical implementations of this approach have been pursued in [30] for statics and in [31] for dynamics. Other $\mathscr{C}^{0}$-implementations of gradient elasticity that have been suggested are based on Padé approximations of Equation (2) [32] and an implementation of the Mindlin theory in which the difference between micro-displacements and macro-displacements is penalised [33].

This paper focusses on gradient elasticity theories for dispersive wave propagation and their subsequent finite element implementation. First, we will briefly revisit in Section 2 a generalised gradient elasticity that includes the terms of Equation (3) as well as the fourth-order time derivative of the displacements. Next, in Section 3 we will focus on four particular versions of the general model that lend themselves to straighforward implementations, 
in particular formats that can be implemented using standard finite elements with $\mathscr{C}^{0}$-continuous interpolation functions. In the next three sections we will study the following properties of the four special gradient elasticity models, namely dispersion properties (Section 4), causality (Section 5) and the variationally consistent boundary conditions (Section 6). Numerical examples are presented in Section 7.

\section{Formulation of the generic model}

In order to keep this contribution self-contained, the formulation of the generic model that includes all fourth-order terms is recapped briefly by revisisting the derivations of Pichugin et al. [26]. Starting point is the simple discrete model depicted in Figure 1. All particles have mass $M$ and all springs have stiffness $K$. Note that we only consider longitudinal displacement - this leads to the gradient elasticity models mentioned above, whereas the inclusion of rotational degrees of freedom would result in Cosserat-type models $[13,34,35]$.

The equation of motion of the central particle reads

$$
M \frac{\partial^{2} u_{n}(t)}{\partial t^{2}}=K\left(u_{n-1}(t)-2 u_{n}(t)+u_{n+1}(t)\right)
$$

where $M$ and $K$ are the particle mass and the spring stiffness, respectively, both of which are assumed to be uniform. The discrete particle displacements $u_{n-1}(t), u_{n}(t)$ and $u_{n+1}(t)$ are replaced by their continuous counterparts $u(x-$ $\ell, t), u(x, t)$ and $u(x+\ell, t)$. Taylor series are applied to $u(x \pm \ell, t)$, by which up to order $O\left(\ell^{2}\right)$ the equations of motion read

$$
\frac{\partial^{2} u(x, t)}{\partial t^{2}}=c_{e}^{2} \frac{\partial^{2} u(x, t)}{\partial x^{2}}+O\left(\ell^{2}\right)
$$

where $c_{e}=\sqrt{E / \rho}$ is the elastic bar velocity, while the mass density $\rho=M / A \ell$, the Young's modulus $E=K \ell / A$ and $A$ is the cross sectional area. A higher-order approximation of Equation (4) is obtained by including the next terms of the Taylor series $[13,14,36]$, that is

$$
\frac{\partial^{2} u(x, t)}{\partial t^{2}}=c_{e}^{2} \frac{\partial^{2} u(x, t)}{\partial x^{2}}+\frac{1}{12} c_{e}^{2} \ell^{2} \frac{\partial^{4} u(x, t)}{\partial x^{4}}+O\left(\ell^{4}\right)
$$

As is well-known, Equation (6) is unstable for wave numbers $k>\sqrt{12} / \ell[16,20,37,38]$ and should not be used to solve boundary value problems [16]. The unstable higher-order term in Equation (6) can be replaced by stable higher-order terms as follows: the second derivative with respect to $x$ is taken of Equation (5); the result is multiplied with $C_{1} \ell^{2}$ and substracted from Equation (6), by which

$$
\frac{\partial^{2} u(x, t)}{\partial t^{2}}-C_{1} \ell^{2} \frac{\partial^{4} u(x, t)}{\partial t^{2} \partial x^{2}}=c_{e}^{2} \frac{\partial^{2} u(x, t)}{\partial x^{2}}-\left(C_{1}-\frac{1}{12}\right) c_{e}^{2} \ell^{2} \frac{\partial^{4} u(x, t)}{\partial x^{4}}+O\left(\ell^{4}\right)
$$

The resulting equation of motion is stable for all wave numbers provided that the model parameter $C_{1}>\frac{1}{12}$. Note that the truncation errors of Equations (6) and (7) are the same, namely $O\left(\ell^{4}\right)$.

Remark 1 The asymptotic equivalence of Equations (6) and (7) has been explored in [37, 38] who used $C_{1}=\frac{1}{12}$. Similarly, in [20,21] a modified relation between the kinematic variables of the discrete model and the continuum model was defined in the transition from Equation (4) to Equation (7).

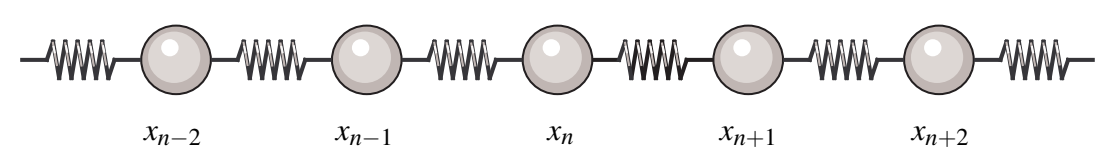

Figure 1: One-dimensional array of particles connected by springs 
Another asymptotically equivalent formulation was obtained by Pichugin et al. [26] by taking the second time derivative of Equation (5) and multiplying the result with $C_{2} \ell^{2} / c_{e}^{2}$. Adding the obtained expression to Equation (7) yields

$$
\begin{array}{r}
\frac{\partial^{2} u(x, t)}{\partial t^{2}}+C_{2} \frac{\ell^{2}}{c_{e}^{2}} \frac{\partial^{4} u(x, t)}{\partial t^{4}}-\left(C_{1}+C_{2}\right) \ell^{2} \frac{\partial^{4} u(x, t)}{\partial t^{2} \partial x^{2}}= \\
c_{e}^{2} \frac{\partial^{2} u(x, t)}{\partial x^{2}}-\left(C_{1}-\frac{1}{12}\right) c_{e}^{2} \ell^{2} \frac{\partial^{4} u(x, t)}{\partial x^{4}}+O\left(\ell^{4}\right)
\end{array}
$$

Equation (8) is yet again asymptotically accurate up to $O\left(\ell^{4}\right)$.

Instead of the particle spacing $\ell$ and the two model parameters $C_{1}$ and $C_{2}$, one can also interpret the coefficients of the higher-order terms as three separate internal length scales. A reformulation of Equation (8) to this extent would read

$$
\frac{\partial^{2} u(x, t)}{\partial t^{2}}-c_{e}^{2} \frac{\partial^{2} u(x, t)}{\partial x^{2}}+\frac{\ell_{1}^{2}}{c_{e}^{2}} \frac{\partial^{4} u(x, t)}{\partial t^{4}}-\ell_{2}^{2} \frac{\partial^{4} u(x, t)}{\partial t^{2} \partial x^{2}}+c_{e}^{2} \ell_{3}^{2} \frac{\partial^{4} u(x, t)}{\partial x^{4}}=0
$$

where $\ell_{1}=\ell \sqrt{C_{2}}, \ell_{2}=\ell \sqrt{C_{1}+C_{2}}$ and $\ell_{3}=\ell \sqrt{C_{1}-\frac{1}{12}}$. While these three internal length scales cannot be chosen independently if the behaviour of Equation (4) is to be approximated, more complicated material models may allow for independent values of the three length scales, see for instance the continualisation of a three-phase material as studied by Metrikine [25].

\section{Formulation of the special models}

It is desirable to have a higher-order elasticity theory that not only describes wave dispersion realistically, but that can also be implemented straightforwardly, i.e. using standard numerical discretisation tools. Here, we will investigate special cases of Equation (9) that lend themselves to straightforward finite element implementations. To this end, fourth-order spatial derivatives require special care, and should ideally be replaced by second-order spatial derivatives. Fourth-order time derivatives can in principle be handled but require an extension of existing numerical time integration schemes.

\subsection{Special model 1: $\ell_{1}=\ell_{3}=0$}

The simplest reduction of Equation (9) that is still dispersive can be obtained by setting $\ell_{1}=0$ (thus avoiding complications in the time integration a priori) as well as $\ell_{3}=0$ (thus avoiding complications with the continuity of the spatial discretisation a priori). The resulting model reads

$$
\frac{\partial^{2} u(x, t)}{\partial t^{2}}-c_{e}^{2} \frac{\partial^{2} u(x, t)}{\partial x^{2}}-\ell_{2}^{2} \frac{\partial^{4} u(x, t)}{\partial t^{2} \partial x^{2}}=0
$$

and this particular format has been suggested by many researchers before in the context of dispersive wave propagation, e.g. [37-40]. The only higher-order term is second-order in space (thus, standard finite element techniques can be used) and second-order in time (this, standard numerical time integration schemes can be used). The dispersive properties of this model are somewhat unusual in that the phase and group velocities of the shorter waves approaches zero and there is a cut-off frequency above which the waves do not propagate (see Section 4). The original model also acts as a low-pass filter that does not propagate waves with frequencies above a cut-off. Therefore, special model 1 can be considered to capture the filtering property of the original model.

\subsection{Special model 2: $\ell_{3}=0$}

As an extension of the previous model, the fourth-order time derivative may be retained. Only the fourth-order spatial derivative is eliminated by setting its coefficient equal to zero, by which

$$
\frac{\partial^{2} u(x, t)}{\partial t^{2}}-c_{e}^{2} \frac{\partial^{2} u(x, t)}{\partial x^{2}}+\frac{\ell_{1}^{2}}{c_{e}^{2}} \frac{\partial^{4} u(x, t)}{\partial t^{4}}-\ell_{2}^{2} \frac{\partial^{4} u(x, t)}{\partial t^{2} \partial x^{2}}=0
$$

The spatial discretisation of this model is straightforward but the time integration algorithm needs adaptations, which are discussed in detail in Appendix B. 
Remark 2 Pichugin et al. [26] argued that two extra orders of accuracy can be gained for Equation (8) through a specific choice for $C_{1}$ and $C_{2}$, namely $C_{1}=\frac{1}{12}$ and $C_{2}=\frac{1}{20}$. With this parameter choice, special model 2 is obtained with $\ell_{1}=\ell / \sqrt{20}$ and $\ell_{2}=\ell \sqrt{2 / 15}$. Note that there is no clear physical meaning of the numerical coefficients $1 / \sqrt{20}$ and $\sqrt{2 / 15}$; they merely follow from exploring the equivalence of two asymptotic series.

Remark 3 Due to the presence of two (rather than one) propagating modes as explained in Section 4, special model 2 may produce high-frequency oscillations that are not present in the original chain-model, especially when the loading contains substantial high-frequency component. As an approximation of Equation (4) this theory is only valid for modelling motions dominated by frequencies below the second mode cut-off.

\subsection{Special model 3: $\ell_{1}=0$}

Another specialisation of Equation (9) is obtained by omitting the fourth-order time derivative but keeping the fourth-order spatial derivative. Thus,

$$
\frac{\partial^{2} u(x, t)}{\partial t^{2}}-c_{e}^{2} \frac{\partial^{2} u(x, t)}{\partial x^{2}}-\ell_{2}^{2} \frac{\partial^{4} u(x, t)}{\partial t^{2} \partial x^{2}}+c_{e}^{2} \ell_{3}^{2} \frac{\partial^{4} u(x, t)}{\partial x^{4}}=0
$$

In fact, this model is the one-dimensional equivalent of Equation (3) and its wave propagation characteristics have been studied before in [2,19-22,24]. The presence of the fourth order spacial derivative means that this model associates two wave numbers to each frequency, one of which does not exist in the original model [26]. This becomes particularly important when formulating boundary conditions for this model.

The development of a finite element implementation of this model has been hampered by the inclusion of the fourth-order spatial derivative. To overcome this, an operator split has been developed by which the fourth-order differential equation (12) is rewritten as a set of two second-order differential equations [18,31], as follows:

- a new displacement-type variable $\hat{u}(x, t)$ is defined through

$$
\hat{u}(x, t)=u(x, t)-\ell_{3}^{2} \frac{\partial^{2} u(x, t)}{\partial x^{2}}
$$

by which Eq. (12) can be rewritten as

$$
\frac{\partial^{2} u(x, t)}{\partial t^{2}}-\ell_{2}^{2} \frac{\partial^{4} u(x, t)}{\partial t^{2} \partial x^{2}}-c_{e}^{2} \frac{\partial^{2} \hat{u}(x, t)}{\partial x^{2}}=0
$$

- Take the second time derivative of Equation (13) and multiply with $\ell_{2}^{2} / \ell_{3}^{2}$. The result is used to replace the second term in Equation (14).

- Take the second time derivative of Equation (13) and multiply with $1-\ell_{2}^{2} / \ell_{3}^{2}$. The result is to be used instead of Equation (13).

- The resulting set of equations is symmetric, fully coupled and contains at most second-order spatial derivatives and at most second-order time derivatives:

$$
\frac{\ell_{2}^{2}}{\ell_{3}^{2}} \frac{\partial^{2} \hat{u}(x, t)}{\partial t^{2}}-\frac{\ell_{2}^{2}-\ell_{3}^{2}}{\ell_{3}^{2}} \frac{\partial^{2} u(x, t)}{\partial t^{2}}-c_{e}^{2} \frac{\partial^{2} \hat{u}(x, t)}{\partial x^{2}}=0
$$

together with

$$
-\frac{\ell_{2}^{2}-\ell_{3}^{2}}{\ell_{3}^{2}} \frac{\partial^{2} \hat{u}(x, t)}{\partial t^{2}}+\frac{\ell_{2}^{2}-\ell_{3}^{2}}{\ell_{3}^{2}} \frac{\partial^{2} u(x, t)}{\partial t^{2}}-\left(\ell_{2}^{2}-\ell_{3}^{2}\right) \frac{\partial^{4} \hat{u}(x, t)}{\partial t^{2} \partial x^{2}}=0
$$

The symmetry issue relates to the coefficient of $u$ in the first equation being equal to the coefficient of $\hat{u}(x, t)$ in the second equation - this facilitates identifying the underlying energy densities and, in turn, natural boundary conditions [31]. 
In the derivations the assumption was made that $\ell_{2} \neq \ell_{3}$. In case $\ell_{2}=\ell_{3}$ a model is obtained that is not dispersive (see also Remark 5 below). The fact that the space and time derivatives are at most of order two means that standard discretisation techniques can be used (e.g. the usual $\mathscr{C}^{0}$-continuous finite elements for spatial discretisation and the standard Newmark scheme for the time integration). From a comparison with Mindlin's 1964 theory the second displacement-type variable $\hat{u}(x, t)$ has been identified as the microscopic displacement whereas the original displacement variable $u(x, t)$ is the macroscopic displacement [41].

\subsection{Special model 4: $\ell_{2}^{2}=\ell_{1}^{2}+\ell_{3}^{2}$}

The case $\ell_{2}^{2}=\ell_{1}^{2}+\ell_{3}^{2}$ allows Equation (9) to be rewritten as

$$
\left(\frac{\partial^{2}}{\partial t^{2}}-c_{e}^{2} \frac{\partial^{2}}{\partial x^{2}}\right)\left(1+\frac{\ell_{1}^{2}}{c_{e}^{2}} \frac{\partial^{2}}{\partial t^{2}}-\ell_{3}^{2} \frac{\partial^{2}}{\partial x^{2}}\right) u(x, t)=0
$$

In going from Equation (9) to Equation (17), all fourth-order derivatives have been factorised as products of secondorder derivatives. Equation (17) can be seen as an operator split in which firstly the equations of classical elasticity are solved, that is

$$
\frac{\partial^{2} u_{c}(x, t)}{\partial t^{2}}-c_{e}^{2} \frac{\partial^{2} u_{c}(x, t)}{\partial x^{2}}=0
$$

where $u_{c}(x, t)$ is the displacement according to classical elasticity. The solution of Equation (18) then serves as the source term for a second equation, i.e.

$$
u(x, t)+\frac{\ell_{1}^{2}}{c_{e}^{2}} \frac{\partial^{2} u(x, t)}{\partial t^{2}}-\ell_{3}^{2} \frac{\partial^{2} u(x, t)}{\partial x^{2}}=u_{c}(x, t)
$$

In contrast to Equations (15-16), the resulting equations of special model 4 are uncoupled: $u(x, t)$ does not appear in Equation (18). Thus, Equations (18) and (19) can be solved sequentially which is less time-consuming than solving the fully coupled system of Equations (15) and (16).

Remark 4 In contrast to the previous 3 special models, special model 4 cannot be obtained from Equation (8) through an appropriate choice for $C_{1}$ and $C_{2}$. Thus, as an approximation of Equation (4) it is only accurate to order $O\left(\ell^{2}\right)$. This can be interpreted physically as follows: the first factor in Equation (17) assumes non-dispersive propagation of the fundamental mode, whereas $O\left(\ell^{4}\right)$ asymptotic accuracy of the dispersion relation of the chain dictates that the fundamental mode must be dispersive [26].

Remark 5 In case $\ell_{1}=0$ it follows that $\ell_{2}=\ell_{3}$. This further particularisation of special model 4 is non-dispersive. This can easily be seen as follows: Equation (18) is non-dispersive, and without time derivatives Equation (19) provides merely a smooting of $u_{c}(x, t)$ in the spatial domain, not in the time domain. Equation (23) in the next Section illustrates this point: the angular frequency would be a linear function of the wave number for such a model.

\section{Dispersion properties}

To investigate the dispersive properties of the various models, a trial solution $u(x, t)=B \exp (\mathrm{i}(k x-\omega t)$ is substituted into Equation (9), whereby $B$ is the amplitude, $k$ is the wave number and $\omega$ is the angular frequency. This results in

$$
\frac{\ell_{1}^{2}}{c_{e}^{2}} \omega^{4}-\left(1+\ell_{2}^{2} k^{2}\right) \omega^{2}+c_{e}^{2} k^{2}\left(1+\ell_{3}^{2} k^{2}\right)=0
$$

For the various models the angular frequency $\omega$ can be resolved as

$$
\text { Model 1: } \quad \omega=c_{e} k \cdot \frac{1}{\sqrt{1+\ell_{2}^{2} k^{2}}}
$$




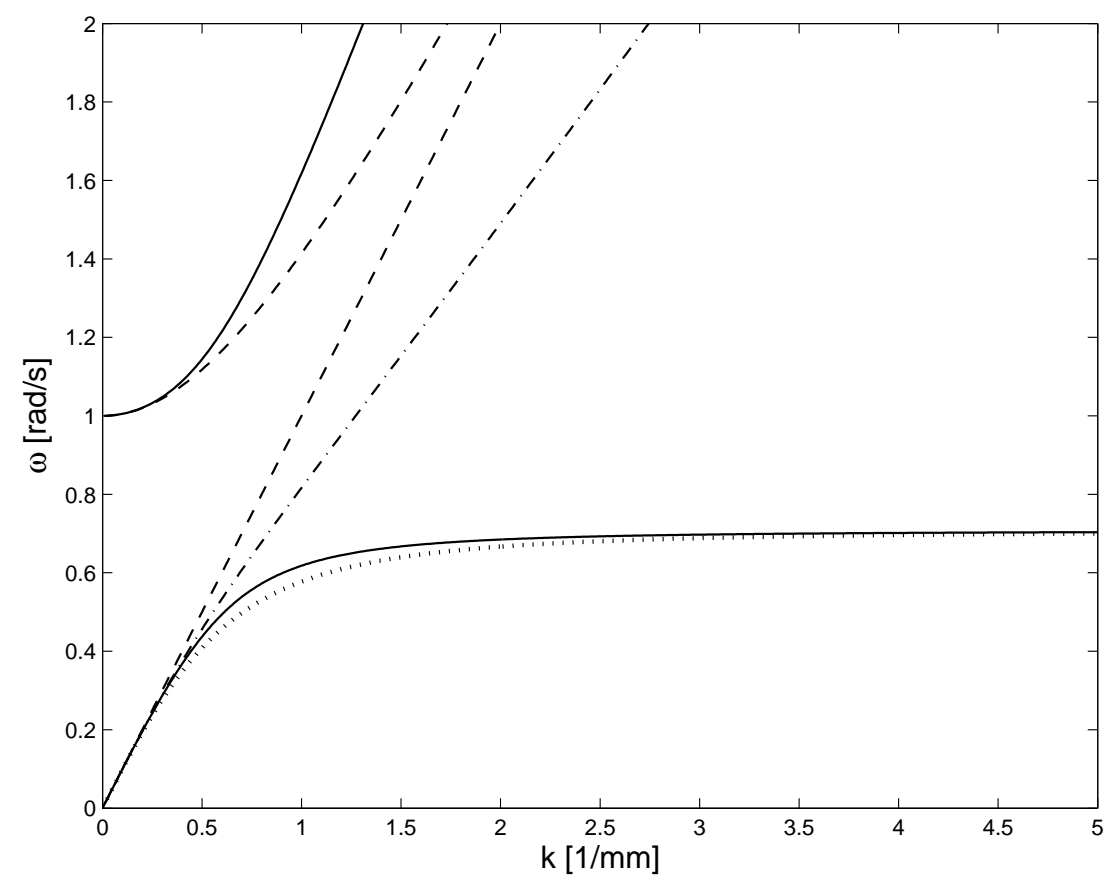

Figure 2: Angular frequency versus wave number for Model 1 (dotted), Model 2 (solid), Model 3 (dash-dotted) and Model 4 (dashed)

$$
\begin{array}{ll}
\text { Model 2: } & \omega=c_{e} k \cdot \sqrt{\frac{1+\ell_{2}^{2} k^{2} \pm \sqrt{\left(1+\ell_{2}^{2} k^{2}\right)^{2}-4 \ell_{1}^{2} k^{2}}}{2 \ell_{1}^{2} k^{2}}} \\
\text { Model 3: } & \omega=c_{e} k \cdot \sqrt{\frac{1+\ell_{3}^{2} k^{2}}{1+\ell_{2}^{2} k^{2}}} \\
\text { Model 4: } & \omega=c_{e} k \quad \bigvee \quad \omega=c_{e} k \cdot \sqrt{\frac{1+\ell_{3}^{2} k^{2}}{\ell_{1}^{2} k^{2}}}
\end{array}
$$

These curves of $\omega$ versus $k$ are plotted in Figure 2 for $c_{e}=1 \mathrm{~m} / \mathrm{s}$ and $\ell_{2}=\sqrt{2} \mathrm{~m}$; furthermore $\ell_{1}=1 \mathrm{~m}$ in Models 2 and 4 and $\ell_{3}=1 \mathrm{~m}$ in Models 3 and 4 . All four models have a primary branch in the $\omega-k$ plane that passes through the origin; these branches were called acoustical branches by Mindlin. In addition, the models with a fourth-order time derivative (i.e. Models 2 and 4) also have a secondary branch that starts at a finite cut-off frequency; Mindlin denoted these the optical branches. Three cases can be distinguished concerning the primary (acoustical) branches:

- the curve attains a horizontal asymptote: this happens in case $\ell_{3}=0$, that is for Models 1 and 2 . The implications are that the phase velocity $c=\omega / k$ will vanish for the larger wave numbers, i.e. the high-frequency waves do not propagate. This mimics the low-pass behaviour of the original problem governed by Equation (4), but may be undesirable in the context of other physical problems. However, this must be judged against the fact that spatial discretisation filters out all frequencies higher than a threshold value set by the discretisation (e.g. by the finite element size).

- the curve attains a non-horizontal asymptote: in case $\ell_{3} \neq 0$ a non-horizontal asymptote is attained for the large wave numbers, the slope of which is governed by the ratio $\ell_{3} / \ell_{2}$. This means that the corresponding phase velocities will be finite for all wave numbers. Such is the case for the present Model 3.

- the curve is a straight line through the origin: for the particular combination of $\ell_{1}, \ell_{2}$ and $\ell_{3}$ of Model 4 the primary branch is given by $\omega=c_{e} k$ which happens to be the same as classical elasticity. As such, the primary branch of Model 4 is associated with the first of the factorised equations, that is expression (18). This particular branch is non-dispersive. 
As regards the secondary (optical) branches, they start at a cut-off frequency given by $\omega=c_{e} / \ell_{1}$, and they attain non-horizontal asymptotes with slopes $c_{e} \ell_{2} / \ell_{1}$ (Model 2) and $c_{e} \ell_{3} / \ell_{1}$ (Model 4). It is noted that the secondary branch of Model 4 corresponds to Equation (19).

\section{Causality}

It is desirable that mechanical models observe the principle of causality [42] according to which the cause has to precede its effect. In fact, all models that are formulated in the time domain do satisfy this principle. The causality may be violated if the frequency-domain formulation is adopted and the Kramers-Kroenig relations [43] are not observed. All the above-introduced models are formulated in the time-space domain and, therefore, satisfy the causality principle.

A stricter requirement than the causality principle is the so-called Einstein's causality that postulates that signals can not propagate faster than the speed of light. In classical mechanics, all disturbances propagate with much lower speeds and Einstein's causality may seem to be of no significance. In our view, however, it is desirable that also classical mechanical models observe Einstein's causality and do not allow signals to propagate energy infinitely fast. In this section, it is investigated whether the above-introduced gradient elasticity models satisfy Einstein's causality.

\subsection{The wave equation}

The wave equation is considered here to demonstrate a method that will be applied to check whether the aboveintroduced gradient elasticity models observe Einstein's causality. The method makes use of the Laplace and Fourier integral transforms that are defined as follows:

$$
u_{s}(x, s)=\int_{0}^{\infty} u(x, t) \exp (-s t) \mathrm{d} t \quad \text { and } \quad u_{s, k}(k, s)=\int_{-\infty}^{\infty} u_{s}(x, s) \exp (\mathrm{i} k x) \mathrm{d} x
$$

Consider the wave equation with the right-hand side representing a point pulse load:

$$
\frac{\partial^{2} u}{\partial t^{2}}-c_{e}^{2} \frac{\partial^{2} u}{\partial x^{2}}=F \delta(x) \delta(t)
$$

where $F$ is a constant and $\delta(\ldots)$ is the Dirac delta-function. Applying the integral transforms defined by Equation (25) to Equation (26), one obtains

$$
u_{s, k}\left(s^{2}+c_{e}^{2} k^{2}\right)=F \quad \Rightarrow \quad u_{s, k}=\frac{F}{s^{2}+c_{e}^{2} k^{2}}
$$

First, the Fourier inversion is applied to Equation (27). Facilitated by the contour integration and the residue theorem [44], it results in

$$
u_{s}=\frac{1}{2 \pi} \int_{-\infty}^{\infty} u_{s, k} \exp (-\mathrm{i} k x) \mathrm{d} k=\frac{F}{2 \pi c_{e}^{2}} \int_{-\infty}^{\infty} \frac{\exp (-\mathrm{i} k x)}{\left(k-\mathrm{i} s / c_{e}\right)\left(k+\mathrm{i} s / c_{e}\right)} \mathrm{d} k=\frac{F}{2 s c_{e}} \exp \left(-\frac{s|x|}{c_{e}}\right)
$$

Next, the inverse Laplace transform [45] is applied:

$$
u(x, t)=\frac{1}{2 \pi \mathrm{i}} \int_{a-\mathrm{i} \infty}^{a+\mathrm{i} \infty} u_{s}(x, s) \exp (s t) \mathrm{d} s
$$

where $a$ is a positive real number that is larger than the real parts of all singularities of $u_{s}(x, s)$. Insertion of Equation (28) into Equation (29) gives

$$
u(x, t)=\frac{F}{4 \pi c_{e} \mathrm{i}} \int_{a-\mathrm{i} \infty}^{a+\mathrm{i} \infty} \frac{1}{s} \exp \left(-\frac{s}{c_{e}}\left(|x|-c_{e} t\right)\right) \mathrm{d} s=\frac{1}{2 \pi \mathrm{i}} \int_{a-\mathrm{i} \infty}^{a+\mathrm{i} \infty} Z(s) \mathrm{d} s
$$



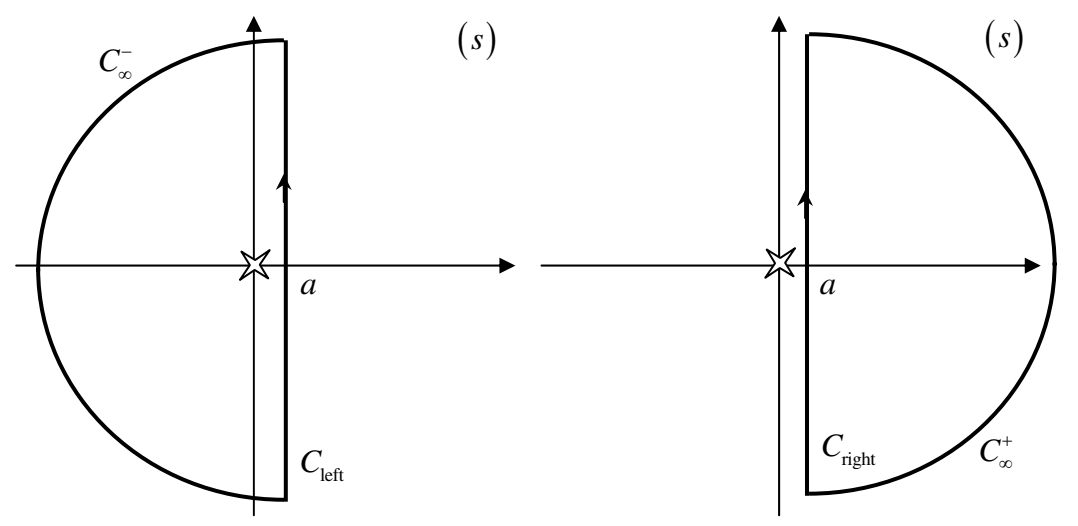

Figure 3: Integration contours in the complex s-plain for $|x|>c_{e} t$ (left) and $|x|<c_{e} t$ (right)

The integral in Equation (30) can be evaluated using the contour integration and the residue theorem. The integration contours are chosen in the manner shown in Figure 3. Let us first consider $|x|<c_{e} t$ and use the contour shown in the left part of Figure 3. According to Cauchy's residue theorem [44], the following holds

$$
\oint_{C_{\mathrm{left}}} Z(s) \mathrm{d} s=\int_{a-\mathrm{i} \infty}^{a+\mathrm{i} \infty} Z(s) \mathrm{d} s+\int_{C_{\infty}^{-}} Z(s) \mathrm{d} s=2 \pi \mathrm{i} \operatorname{Res}_{s=0}(Z(s))
$$

where $\operatorname{Res}_{s=0}(Z(s))$ is the residue of $Z(s)$ at $s=0$. Due to Jordan's lemma [44], the integral over the semicircle $C_{\infty}^{-}$ vanishes as its radius tends to infinity. Therefore,

$$
u(x, t)_{|x|<c_{e} t}=\frac{1}{2 \pi \mathrm{i}} \int_{a-\mathrm{i} \infty}^{a+\mathrm{i} \infty} Z(s) \mathrm{d} s=\operatorname{Res}_{s=0}(Z(s))=\frac{F}{2 c_{e}}
$$

For $|x|>c_{e} t$, the contour shown in the right part of Figure 3 can be used. As this contour surrounds no singularity, Cauchy's residue theorem gives

$$
\oint_{C_{\text {right }}} Z(s) \mathrm{d} s=\int_{a-\mathrm{i} \infty}^{a+\mathrm{i} \infty} Z(s) \mathrm{d} s+\int_{C_{\infty}^{+}} Z(s) \mathrm{d} s=0
$$

Due to Jordan's lemma, the integral over the infinite semicircle $C_{\infty}^{+}$is zero and, therefore,

$$
u(x, t)_{|x|>c_{e} t}=\frac{1}{2 \pi \mathrm{i}} \int_{a-\mathrm{i} \infty}^{a+\mathrm{i} \infty} Z(s) \mathrm{d} s=0
$$

Equations (32) and (34) reproduce a well-known result that propagation of disturbances in a medium described by the one-dimensional wave equation is bounded by two propagating fronts located at $|x|=c_{e} t$. This result is reproduced here to show that existence of the fronts (and, consequently, Einstein's causality) can be concluded upon by a relatively simple analysis of the argument of the exponent in the inverse Laplace transform, Equation (30). Indeed, the fronts can be associated with the transition of the contour closure (the semicircles in Figure 3) from the left half-plane of the complex s-plain to its right half-plane. This transition, in turn, is associated with the possibility to close the contour such that the integral along the closure vanishes as its radius tends to infinity. The latter is one-to-one related to the argument of the exponent in the inverse Laplace transform. If in the limit $\operatorname{Re}(s) \rightarrow+\infty$ the real part of this argument tends to minus infinity for $|x|>C t, C>0$ then the fronts exist at $|x|=C t$ and Einsteins causality is satisfied. This criterion will be used in the following subsections to conclude on Einstein's causality of special models $1-4$. 


\subsection{Special model 1}

Application of the integral transforms defined by Equation (25) to Equation (10) enriched by $F \delta(x) \delta(t)$ on the right-hand side, results in

$$
u_{s, k}\left(s^{2}+c_{e}^{2} k^{2}+\ell_{2}^{2} s^{2} k^{2}\right)=F \quad \Rightarrow \quad u_{s, k}=\frac{F}{s^{2}+c_{e}^{2} k^{2}+\ell_{2}^{2} s^{2} k^{2}}
$$

The inverse Fourier transform is accomplished as follows

$$
u_{s}=\frac{F}{2 \pi} \int_{-\infty}^{\infty} \frac{\exp (-\mathrm{ikx})}{s^{2}+c_{e}^{2} k^{2}+\ell_{2}^{2} s^{2} k^{2}} \mathrm{~d} k=\frac{F}{2 \pi\left(c_{e}^{2}+\ell_{2}^{2} s^{2}\right)} \int_{-\infty}^{\infty} \frac{\exp (-\mathrm{i} k x)}{\left(k-k_{1}\right)\left(k-k_{2}\right)} \mathrm{d} k
$$

where

$$
k_{1}=\frac{\text { is }}{\sqrt{c_{e}^{2}+\ell_{2}^{2} s^{2}}} \quad \text { and } \quad k_{2}=\frac{-\mathrm{i} s}{\sqrt{c_{e}^{2}+\ell_{2}^{2} s^{2}}}
$$

Choosing the branch of the square root in Equation (37) such that the real part of this root is positive in the whole $s$-plane, one can use the contour integration and evaluate Equation (36) as

$$
u_{s}=-2 \pi \mathrm{i} \frac{F}{2 \pi\left(c_{e}^{2}+\ell_{2}^{2} s^{2}\right)} \frac{\exp \left(-\mathrm{i} k_{2}|x|\right)}{\left(k_{2}-k_{1}\right)}=\frac{1}{2 s \sqrt{c_{e}^{2}+\ell_{2}^{2} s^{2}}} \exp \left(-\frac{s|x|}{\sqrt{c_{e}^{2}+\ell_{2}^{2} s^{2}}}\right)
$$

Application of the inverse Laplace transform to Equation (38) gives

$$
u(x, t)=\frac{F}{4 \pi \mathrm{i}} \int_{a-\mathrm{i} \infty}^{a+\mathrm{i} \infty} \frac{1}{s \sqrt{c_{e}^{2}+\ell_{2}^{2} s^{2}}} \exp \left(\frac{-s|x|}{\sqrt{c_{e}^{2}+\ell_{2}^{2} s^{2}}}+s t\right) \mathrm{d} s
$$

In the limit $\operatorname{Re}(s) \rightarrow+\infty$ the argument of the exponent in Equation (39) tends to $-|x| / \ell_{2}+s t$ which makes it clear that the integral over the infinite semicircle in the right half-plane of the complex $s$-plane does not vanish for any $x$. This means that there are no fronts in model 1 and this model does not observe Einstein's causality.

Note that the Laplace solution given by Equation (38) differs significantly from that given by Equation (28). The difference consists in the existence of the branch points and branch cuts in the complex $s$-plane that are associated with the chosen branch of the square root in Equation (38). However, as the branch points and the branch cuts are located at the left of the inverse-Laplace integration contour (the straight vertical line in Figure 3), the criterion based on the argument of the exponent remains applicable.

\subsection{Special model 2}

The Laplace-Fourier solution of Equation (11) that is subjected to $F \delta(x) \delta(t)$ at its right-hand side reads

$$
u_{s, k}=\frac{F}{s^{2}+c_{e}^{2} k^{2}+\ell_{1}^{2} s^{4} / c_{e}^{2}+\ell_{2}^{2} s^{2} k^{2}}
$$

The Fourier inversion can be accomplished in the same manner as for model 1 to give

$$
u_{s}=\frac{1}{2 s \sqrt{c_{e}^{2}+\ell_{2}^{2} s^{2}} \sqrt{1+\ell_{1}^{2} s^{4} / c_{e}^{2}}} \exp \left(-s|x| \frac{\sqrt{1+\ell_{1}^{2} s^{4} / c_{e}^{2}}}{\sqrt{c_{e}^{2}+\ell_{2}^{2} s^{2}}}\right)
$$

where the branches of the square roots are chosen such that the real parts of both roots are positive in the whole $s$-plane. In the limit $\operatorname{Re}(s) \rightarrow+\infty$ the argument of the exponent in the inverse Laplace transform of Equation (41) reads

$$
\operatorname{Arg}_{\infty}=\left(s t-s|x| \frac{\sqrt{1+\ell_{1}^{2} s^{4} / c_{e}^{2}}}{\sqrt{c_{e}^{2}+\ell_{2}^{2} s^{2}}}\right)_{\operatorname{Re}(s) \rightarrow+\infty}=-\frac{s}{c_{e}}\left(|x| \frac{\ell_{1}}{\ell_{2}}-c_{e} t\right)_{\operatorname{Re}(s) \rightarrow+\infty}
$$

The above argument tends to minus infinity provided that $|x|>c_{e} t \ell_{2} / \ell_{1}$. Thus, this model observes Einstein's causality as it predicts signals to propagate no faster than with the speed $c_{e} \ell_{2} / \ell_{1}$. 


\subsection{Special model 3}

The Laplace-Fourier solution for this model is given as

$$
u_{s, k}=\frac{F}{s^{2}+c_{e}^{2} k^{2}+\ell_{2}^{2} s^{2} k^{2}+\ell_{3}^{2} c_{e}^{2} k^{4}}
$$

In contrast to the preceding models, the denominator of this solution has four $k$-roots. Let us write the expressions for these roots directly in the limit $\operatorname{Re}(s) \rightarrow+\infty$. In this limit

$$
k_{1}=\frac{\mathrm{i}}{\ell_{2}}, \quad k_{2}=-\frac{\mathrm{i}}{\ell_{2}}, \quad k_{3}=\mathrm{i} \frac{s}{c_{e}} \frac{\ell_{2}}{\ell_{3}} \quad \text { and } \quad k_{4}=-\mathrm{i} \frac{s}{c_{e}} \frac{\ell_{2}}{\ell_{3}}
$$

Accordingly, the inverse Fourier transform of Equation (43) in this limit can be evaluated as

$$
\begin{aligned}
u_{s} & =\frac{F}{2 \pi \ell_{3}^{2} c_{e}^{2}} \int_{-\infty}^{\infty} \frac{\exp (-\mathrm{i} k x)}{\left(k-k_{1}\right)\left(k-k_{2}\right)\left(k-k_{3}\right)\left(k-k_{4}\right)} \mathrm{d} k \\
& =-\frac{\mathrm{i} F}{\ell_{3}^{2} c_{e}^{2}}\left(\frac{\exp \left(-\mathrm{i} k_{2}|x|\right)}{\left(k_{2}-k_{1}\right)\left(k_{2}-k_{3}\right)\left(k_{2}-k_{4}\right)}+\frac{\exp \left(-\mathrm{i} k_{4}|x|\right)}{\left(k_{4}-k_{1}\right)\left(k_{4}-k_{2}\right)\left(k_{4}-k_{3}\right)}\right) \\
\stackrel{\operatorname{Re}(s) \rightarrow+\infty}{=} & \frac{1}{2} \frac{F}{s^{2} \ell_{2}} \exp \left(-\frac{|x|}{l_{2}}\right)-\frac{1}{2} \frac{F c_{e} \ell_{3}}{s^{3} \ell_{2}^{3}} \exp \left(-\frac{s|x| \ell_{3}}{c_{e}} \frac{\ell_{2}}{\ell_{2}}\right)
\end{aligned}
$$

Thus, the inverse Laplace transform should be taken of the two terms, which correspond to the following arguments of the exponents in the limit $\operatorname{Re}(s) \rightarrow+\infty$ :

$$
\operatorname{Arg} 1_{\infty}=s t-\frac{|x|}{\ell_{2}} \quad \text { and } \quad \operatorname{Arg} 2_{\infty}=-\frac{s}{c_{e}}\left(|x| \frac{\ell_{3}}{\ell_{2}}-c_{e} t\right)
$$

Although $\operatorname{Arg} 2_{\infty}$ ensures a certain abrupt change at $|x|=c_{e} t \ell_{2} / \ell_{3}, \operatorname{Arg} 1_{\infty}$ does not tend to minus infinity as $\operatorname{Re}(s) \rightarrow$ $+\infty$. Therefore, this model allows signals to spread infinitely fast and, consequently, it does not observe Einstein's causality.

\subsection{Special model 4}

The Laplace-Fourier solution for this model is given as

$$
u_{s, k}=\frac{F}{\left(s^{2}+c_{e}^{2} k^{2}\right)\left(1+\ell_{1}^{2} s^{2} / c_{e}^{2}+\ell_{3}^{2} k^{2}\right)}
$$

The $k$-roots of the denominator in the limit $\operatorname{Re}(s) \rightarrow+\infty$ read

$$
k_{1}=\frac{\mathrm{i} s}{c_{e}}, \quad k_{2}=-\frac{\mathrm{i} s}{c_{e}}, \quad k_{3}=\frac{\mathrm{i} s}{c_{e}} \frac{\ell_{1}}{\ell_{3}} \quad \text { and } \quad k_{4}=-\frac{\mathrm{i} s}{c_{e}} \frac{\ell_{1}}{\ell_{3}}
$$

Accordingly, the inverse Fourier transform of Equation (47) in the limit $\operatorname{Re}(s) \rightarrow+\infty$ can be evaluated as

$$
\begin{aligned}
u_{s} & =\frac{F}{2 \pi \ell_{3}^{2} c_{e}^{2}} \int_{-\infty}^{\infty} \frac{\exp (-\mathrm{i} k x)}{\left(k-k_{1}\right)\left(k-k_{2}\right)\left(k-k_{3}\right)\left(k-k_{4}\right)} \mathrm{d} k \\
& =-\frac{\mathrm{i} F}{\ell_{3}^{2} c_{e}^{2}}\left(\frac{\exp \left(-\mathrm{i} k_{2}|x|\right)}{\left(k_{2}-k_{1}\right)\left(k_{2}-k_{3}\right)\left(k_{2}-k_{4}\right)}+\frac{\exp \left(-\mathrm{i} k_{4}|x|\right)}{\left(k_{4}-k_{1}\right)\left(k_{4}-k_{2}\right)\left(k_{4}-k_{3}\right)}\right) \\
\stackrel{\operatorname{Re}(s) \rightarrow+\infty}{=} & -\frac{1}{2} \frac{F c_{e}}{s^{3}\left(\ell_{3}^{2}-\ell_{1}^{2}\right)} \exp \left(-\frac{s|x|}{c_{e}}\right)+\frac{1}{2} \frac{F c_{e} \ell_{3}}{s^{3} \ell_{1}\left(\ell_{3}^{2}-\ell_{1}^{2}\right)} \exp \left(-\frac{s|x|}{c_{e}} \frac{\ell_{1}}{\ell_{3}}\right)
\end{aligned}
$$

The arguments of the exponents in the inverse Laplace transform, as follows from Equation (49) with $\operatorname{Re}(s) \rightarrow+\infty$, have the following form:

$$
\operatorname{Arg} 1_{\infty}=-\frac{s}{c_{e}}\left(|x|-c_{e} t\right), \quad \operatorname{Arg} 2_{\infty}=-\frac{s}{c_{e}}\left(|x| \frac{\ell_{1}}{\ell_{3}}-c_{e} t\right)
$$

As $\operatorname{Re}(s) \rightarrow+\infty$, the first and the second arguments tend to minus infinity at $|x|>c_{e} t$ and $|x|>c_{e} t \ell_{3} / \ell_{1}$, respectively. Thus, as for the physical validity of this model $\ell_{3} / \ell_{1}$ must be not smaller than unity (see the dispersion analysis of Section 4), the signals are always confined within the leading fronts $|x|=c_{e} t \ell_{3} / \ell_{1}$ and the model observes Einstein's causality. 


\section{Boundary conditions}

In order to formulate the consistent boundary conditions of the various models, a variational approach will be taken that later on straigtforwardly leads to the associated finite element equations. Assuming a domain $x \in[0, L]$, the weak form of Equation (9) reads

$$
\begin{array}{r}
\int_{0}^{L} w(x)\left(\frac{\partial^{2} u(x, t)}{\partial t^{2}}+\frac{\ell_{1}^{2}}{c_{e}^{2}} \frac{\partial^{4} u(x, t)}{\partial t^{4}}\right) \mathrm{d} x+\int_{0}^{L} \frac{\partial w(x)}{\partial x}\left(c_{e}^{2} \frac{\partial u(x, t)}{\partial x}+\ell_{2}^{2} \frac{\partial^{3} u(x, t)}{\partial t^{2} \partial x}\right) \mathrm{d} x \\
+\int_{0}^{L} \frac{\partial^{2} w(x)}{\partial x^{2}} c_{e}^{2} \ell_{3}^{2} \frac{\partial^{2} u(x, t)}{\partial x^{2}} \mathrm{~d} x= \\
{\left[w(x)\left(c_{e}^{2} \frac{\partial u(x, t)}{\partial x}+\ell_{2}^{2} \frac{\partial^{3} u(x, t)}{\partial t^{2} \partial x}-\ell_{3}^{2} c_{e}^{2} \frac{\partial^{3} u(x, t)}{\partial x^{3}}\right)\right]_{0}^{L}+\left[\frac{\partial w(x)}{\partial x} \ell_{3}^{2} c_{e}^{2} \frac{\partial^{2} u(x, t)}{\partial x^{2}}\right]_{0}^{L}}
\end{array}
$$

where $w(x)$ is an appropriate test function and integration by parts has been applied once and twice for terms with first-order spatial derivatives and second-order spatial derivatives, respectively. The right-hand-side of Equation (51) reveals the format of the boundary conditions, namely

$$
\begin{array}{cl}
\text { either prescribe } u(x, t) & \text { or prescribe } c_{e}^{2}\left(\frac{\partial u(x, t)}{\partial x}-\ell_{3}^{2} \frac{\partial^{3} u(x, t)}{\partial x^{3}}\right)+\ell_{2}^{2} \frac{\partial^{3} u(x, t)}{\partial t^{2} \partial x} \\
\text { either prescribe } \frac{\partial u(x, t)}{\partial x} & \text { or prescribe } \ell_{3}^{2} c_{e}^{2} \frac{\partial^{2} u(x, t)}{\partial x^{2}}
\end{array}
$$

Note that the natural boundary conditions can be expressed in terms of stress-type variables, that is a Cauchy stress $\sigma$ as

$$
\sigma=E\left(\varepsilon-\ell_{3}^{2} \frac{\partial^{2} \varepsilon}{\partial x^{2}}\right)+\rho \ell_{2}^{2} \frac{\partial^{2} \varepsilon}{\partial t^{2}}
$$

where $\varepsilon=\partial u(x, t) / \partial t$ is the uniaxial strain, and a higher-order stress $\tau$ as

$$
\tau=E \ell_{3}^{2} \frac{\partial \varepsilon}{\partial x}
$$

As regards the non-standard higher-order boundary conditions, most researchers employ homogeneous natural boundary conditions, that is setting $\tau=0$. Essential boundaries were analysed in [46], where it was shown that the boundary conditions of expressions (52) and (53) may result in $O\left(\ell^{2}\right)$ error caused by the presense of boundary layers in theories with higher-order spacial derivatives. An asymptotic procedure that can reduce this error below $O\left(\ell^{3}\right)$ is also suggested in [46].

\subsection{Special models 1 and 2}

Special models 1 and 2 are retrieved from the original model by a specific choice of parameters, without further manipulations. Thus, the boundary conditions for these two models can be retrieved as well from the boundary conditions of the original model. Note that there is only one essential boundary condition and only one natural boundary conditions in these two models, that is, expression (53) does not apply.

\subsection{Special model 3}

In the formulation of special model 3, a few manipulations have been made by which the boundary conditions are affected. To find their appropriate format, the weak form of Equations (15-16) is taken, that is

$$
\int_{0}^{L} \hat{w}(x)\left(\frac{\ell_{2}^{2}}{\ell_{3}^{2}} \frac{\partial^{2} \hat{u}(x, t)}{\partial t^{2}}-\frac{\ell_{2}^{2}-\ell_{3}^{2}}{\ell_{3}^{2}} \frac{\partial^{2} u(x, t)}{\partial t^{2}}\right) \mathrm{d} x+\int_{0}^{L} \frac{\partial \hat{w}(x)}{\partial x} c_{e}^{2} \frac{\partial \hat{u}(x, t)}{\partial x} \mathrm{~d} x=\left[\hat{w}(x) c_{e}^{2} \frac{\partial \hat{u}(x, t)}{\partial x}\right]_{0}^{L}
$$


and

$$
\begin{array}{r}
\int_{0}^{L} w(x)\left(-\frac{\ell_{2}^{2}-\ell_{3}^{2}}{\ell_{3}^{2}} \frac{\partial^{2} \hat{u}(x, t)}{\partial t^{2}}+\frac{\ell_{2}^{2}-\ell_{3}^{2}}{\ell_{3}^{2}} \frac{\partial^{2} u(x, t)}{\partial t^{2}}\right) \mathrm{d} x+\int_{0}^{L} \frac{\partial w(x)}{\partial x}\left(\ell_{2}^{2}-\ell_{3}^{2}\right) \frac{\partial^{3} \hat{u}(x, t)}{\partial t^{2} \partial x} \mathrm{~d} x \\
=\left[w(x)\left(\ell_{2}^{2}-\ell_{3}^{2}\right) \frac{\partial^{3} \hat{u}(x, t)}{\partial t^{2} \partial x}\right]_{0}^{L}
\end{array}
$$

where again integration by parts has been applied to terms with spatial derivatives. From the right-hand-sides of Equations (56) and (57) the boundary conditions are found as

$$
\begin{array}{ll}
\text { either prescribe } \hat{u}(x, t) & \text { or prescribe } c_{e}^{2} \frac{\partial \hat{u}(x, t)}{\partial x}=c_{e}^{2}\left(\frac{\partial u(x, t)}{\partial x}-\ell_{3}^{2} \frac{\partial^{3} u(x, t)}{\partial x^{3}}\right) \\
\text { either prescribe } u(x, t) & \text { or prescribe }\left(\ell_{2}^{2}-\ell_{3}^{2}\right) \frac{\partial^{3} u(x, t)}{\partial t^{2} \partial x}
\end{array}
$$

Note that the two natural boundary conditions of special model 3 together constitute the Cauchy stress as given in Equation (54), albeit with a different factor accompanying the higher-order inertia term.

Although the model is derived from a format that includes a fourth-order spatial derivative, there are no higherorder stresses present in this model and expression (53) does not apply. However, it is possible to emulate the effects of the higher-order stresses by setting a tying between the degrees of freedom $\hat{u}$ and $u$. That is, by setting $\hat{u}(x, t)=u(x, t)$ on the boundary it is implicitly set through Equation (13) that

$$
\ell_{3}^{2} \frac{\partial^{2} u(x, t)}{\partial x^{2}}=0
$$

on the boundary, which is equivalent to setting the higher-order stress $\tau$ equal to zero on the boundary.

\subsection{Special model 4}

In special model 4, the mechanical equations are treated in two consecutive steps. Firstly, Equation (18) is solved and it is accompanied by the usual boundary conditions of classical elasticity, that is

$$
\text { either prescribe } u_{c}(x, t) \quad \text { or prescribe } c_{e}^{2} \frac{\partial u_{c}(x, t)}{\partial x}
$$

To retrieve the boundary conditions corresponding to the second step, the weak form of Equation (19) is taken. Integration by parts of the term with spatial derivatives yields

$$
\int_{0}^{L} w(x)\left(u(x, t)+\frac{\ell_{1}^{2}}{c_{e}^{2}} \frac{\partial^{2} u(x, t)}{\partial t^{2}}\right) \mathrm{d} x+\int_{0}^{L} \frac{\partial w(x)}{\partial x} \ell_{3}^{2} \frac{\partial u(x, t)}{\partial x} \mathrm{~d} x=\left[w(x) \ell_{3}^{2} \frac{\partial u(x, t)}{\partial x}\right]_{0}^{L}+\int_{0}^{L} w(x) u_{c}(x, t) \mathrm{d} x
$$

so that the boundary conditions are found as

$$
\text { either prescribe } u(x, t) \quad \text { or prescribe } \ell_{3}^{2} \frac{\partial u(x, t)}{\partial x}
$$

This model is the only special model to retain all terms that appear in the original model of Equation (9). It is thus of interest to compare the boundary conditions given through expressions (52-53) with those in expressions (61) and (63). The first essential and natural boundary conditions in both models are stated in terms of a displacement and a Cauchy-type stress. However, the second essential boundary condition contains a strain-type variable in expression (53) in contrast to the displacement variable in expression (63). Similarly, the second natural boundary condition contains a higher-order stress in expression (53) in contrast to a strain-type variable in expression (63).

Clearly, there is a mismatch between the two sets of boundary conditions. A simple way to amend this is to take the spatial derivative of Equation (19), that is

$$
\varepsilon(x, t)+\frac{\ell_{1}^{2}}{c_{e}^{2}} \frac{\partial^{2} \varepsilon(x, t)}{\partial t^{2}}-\ell_{3}^{2} \frac{\partial^{2} \varepsilon(x, t)}{\partial x^{2}}=\frac{\partial u_{c}(x, t)}{\partial x}
$$

in which the strain $\varepsilon$ is the primary unknown. The boundary conditions accompanying Equation (64) are

$$
\text { either prescribe } \varepsilon(x, t) \quad \text { or prescribe } \ell_{3}^{2} \frac{\partial \varepsilon(x, t)}{\partial x}
$$

which are compatible with those given in expression (53). 


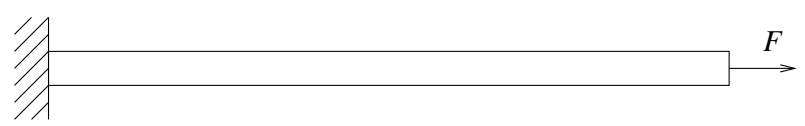

Figure 4: One-dimensional dynamic bar problem — geometry and loading conditions
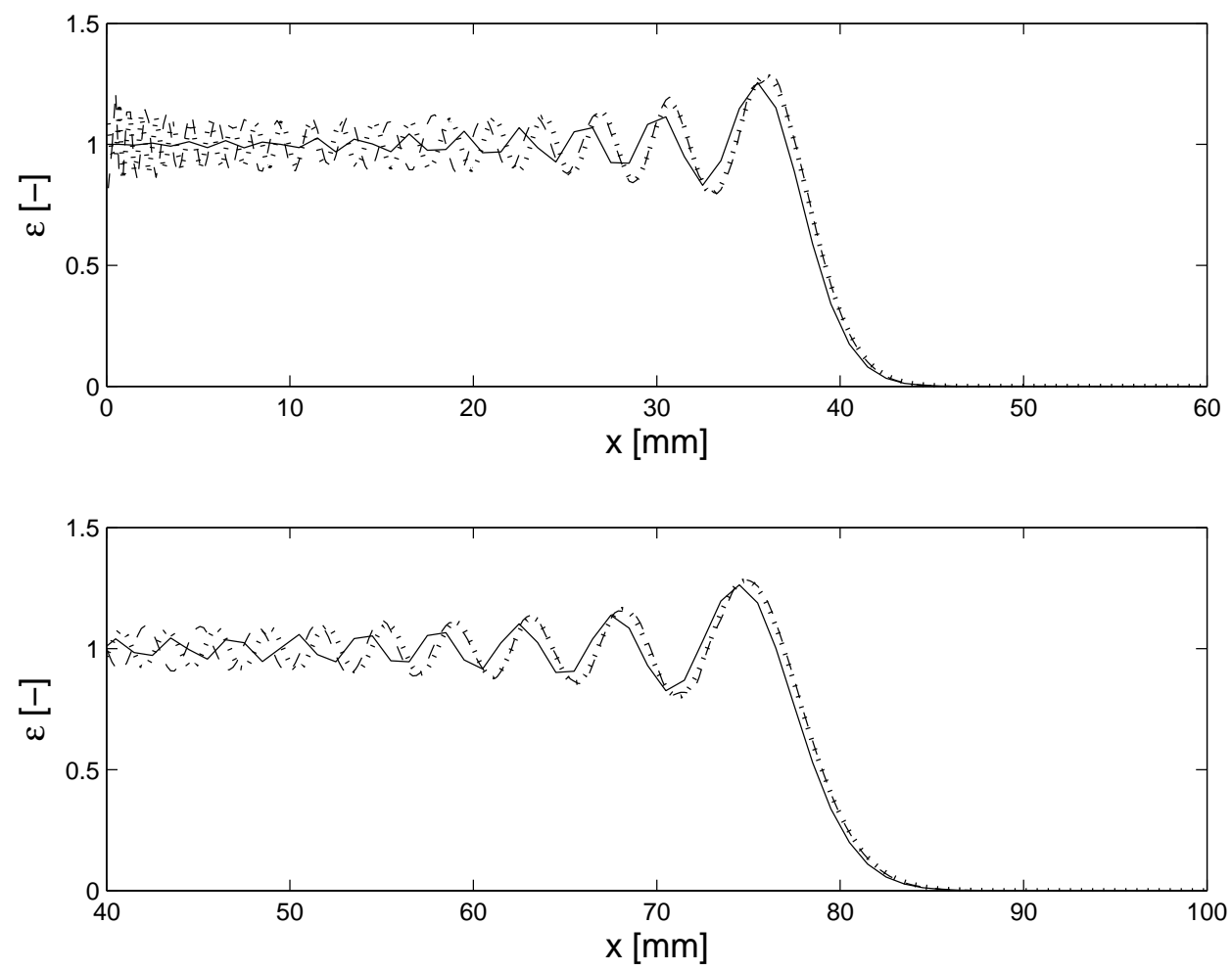

Figure 5: Wave propagation simulated with discrete model (solid), model 1 (dotted) and model 2 (dashed) at time instants $40 \mathrm{~s}$ (top) and $80 \mathrm{~s}$ (bottom)

\section{Examples}

In this Section we present a number of results for the different models. The test set-up is depicted in Figure 4, whereby the length of the bar $L=100 \mathrm{~mm}$. A Heaviside forcing function $F$ of magnitude $1 \mathrm{~N}$ was applied. The values of the internal length scales are specified for each particular example but are taken in the order of magnitude of $1 \mathrm{~mm}$. In order for numerical dispersion to be negligible compared to material dispersion, the bar is discretised with 500 linear finite elements and the time step size is $\Delta t=0.2 \mathrm{~s}$ in the time integration - this concurs with the recommendations for special model 1 given in [47]. The finite element equations for each special model are given in Appendix A.

\subsection{Comparison of special models 1 and 2 with discrete model}

Equations (7) and (8) have been suggested as approximations of Equation (4). It is thus of interest to investigate to which extent the behaviour of Equation (4) can be captured using the gradient elasticity theories. Special model 1, obtained by setting $C_{1}=\frac{1}{12}$ and $C_{2}=0$, has been proposed in earlier studies [37,38,40]. Pichugin et al. argued that optimal parameter choices are given by $C_{1}=\frac{1}{12}$ and $C_{2}=\frac{1}{20}$, see [26]. With these parameters, special model 2 is obtained.

An exact solution for the acceleration of particle $n$ in the discrete model according to Equation (4) has been 

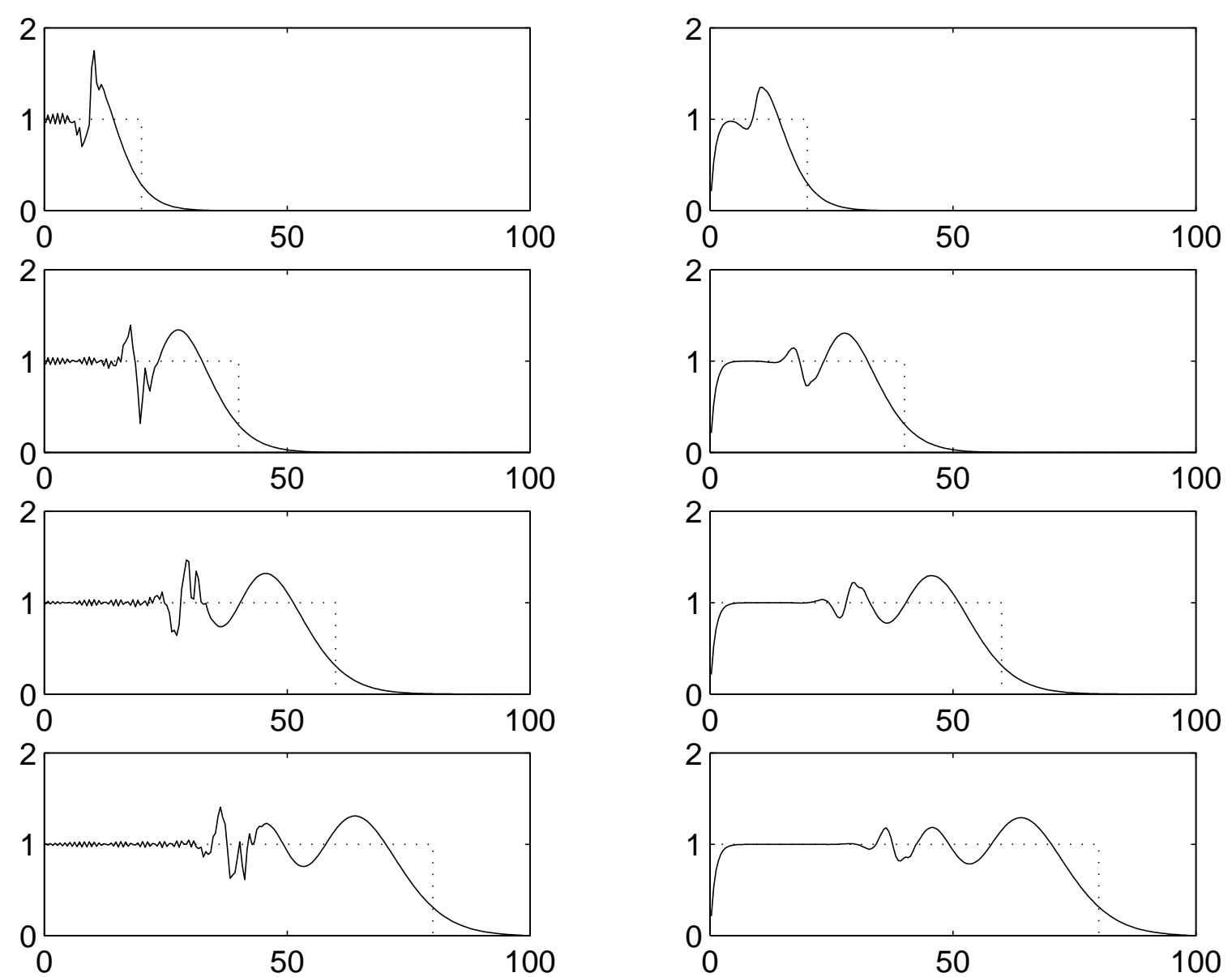

Figure 6: Wave propagation simulated with model 3 - microscopic strain (left) and macroscopic strain (right) across bar for times $20 \mathrm{~s}, 40 \mathrm{~s}, 60 \mathrm{~s}$ and $80 \mathrm{~s}$. Boundary conditions have not been corrected. Dotted lines indicate the analytical solution for classical elasticity.

given in [48] and reads

$$
a_{n}(t)=\frac{2 n-1}{t} J_{2 n-1}(2 t)
$$

where $J$ is the Bessel function of the first kind of order $2 n-1$. In Figure 5 this exact solution is compared to the solutions of special models 1 and 2 for time instants $40 \mathrm{~s}$ and $80 \mathrm{~s}$, whereby we have taken a particle spacing $\ell=1 \mathrm{~mm}$. Thus, $\ell_{1}=0$ and $\ell_{2}=1 / \sqrt{12} \mathrm{~mm}$ for model 1 , whereas for model 2 we have $\ell_{1}=1 / \sqrt{20} \mathrm{~mm}$ and $\ell_{2}=\sqrt{2 / 15} \mathrm{~mm}$. Generally, there is a good correspondence between the three solutions, especially around the wave front. Compared to special model 1, special model 2 captures the slow travelling higher frequencies of the discrete model somewhat better.

\subsection{Influence of boundary conditions in models 3 and 4}

Next, the importance of using homogeneous higher-order natural boundary conditions is demonstrated for special models 3 and 4. Whereas models 1 and 2 are found from the original model of Equation (9) through a suitable selection of values for the various length scales, models 3 and 4 are obtained through a number of mathematical manipulations. As a result, the natural consistent boundary conditions of models 3 and 4 are different from those of the original model, although amendments have been suggested in Section 6. These boundary conditions and their suggested improvement are studied in the same problem as above, whereby the length of the bar $L=100$ $\mathrm{m}$, Young's modulus $E=1 \mathrm{~N} / \mathrm{m}^{2}$ and mass density $\rho=1 \mathrm{~kg} / \mathrm{m}^{3}$. The length scales $\ell_{2}=2 \mathrm{~m}$ and $\ell_{3}=1 \mathrm{~m}$ for 

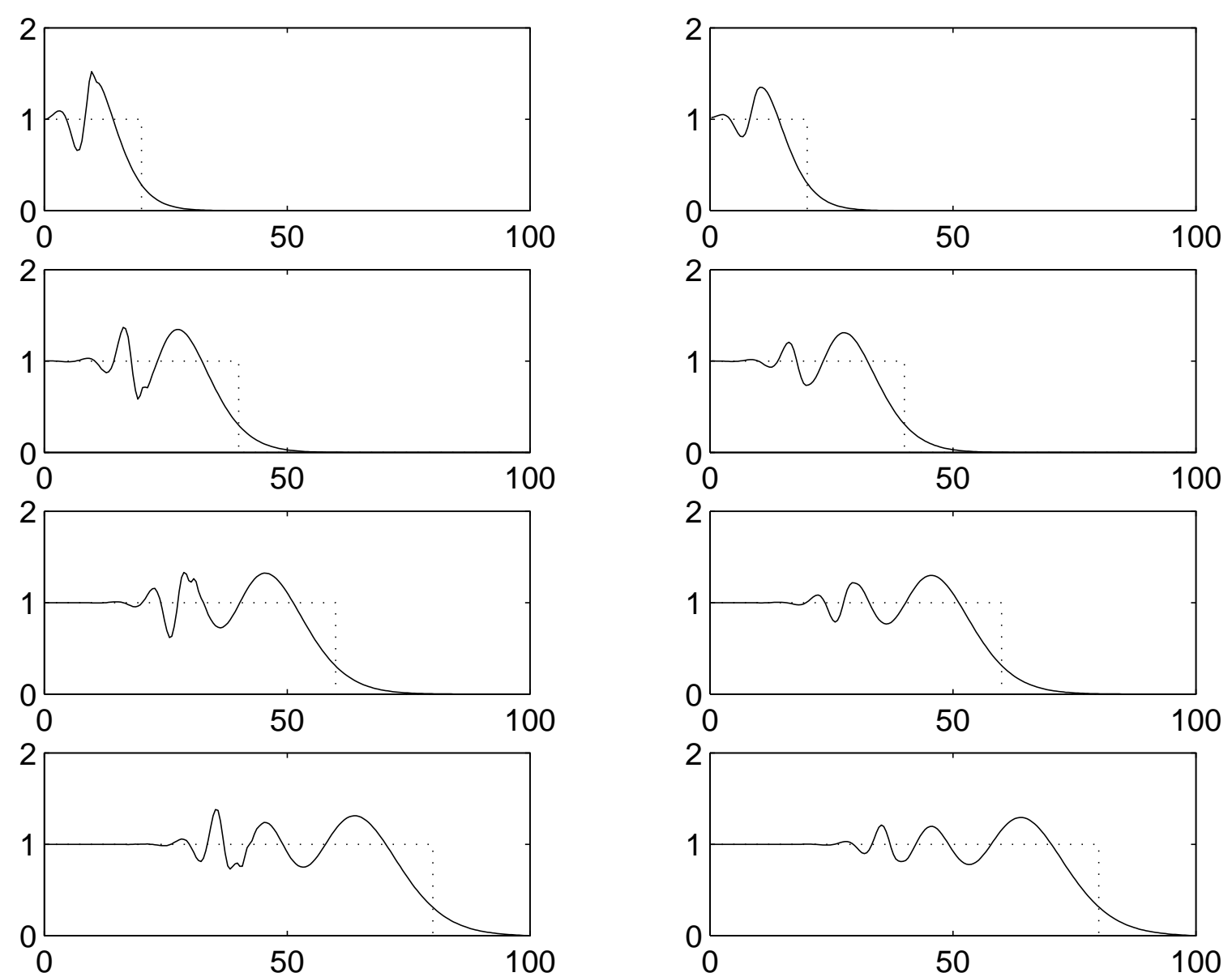

Figure 7: Wave propagation simulated with model 3 - microscopic strain (left) and macroscopic strain (right) across bar for times $20 \mathrm{~s}, 40 \mathrm{~s}, 60 \mathrm{~s}$ and $80 \mathrm{~s}$. Boundary conditions have been corrected by setting $\hat{u}=u$. Dotted lines indicate the analytical solution for classical elasticity.

model 3, while $\ell_{1}=\ell_{3}=1 \mathrm{~m}$ for model 4 . The bar is discretised with 200 linear finite elements and for the time integration a time step size of $0.5 \mathrm{~s}$ is used.

Firstly, special model 3 is considered. At the free end, the natural boundary conditions of expressions (58) and (59) are used, that is $\partial \hat{u} / \partial x=1$ and $\partial^{3} u / \partial t^{2} \partial x=0$. It was suggested in Section 6 to emulate zero higherorder stresses on the boundary by setting a relation between the microscopic displacement $\hat{u}$ and the macroscopic displacement $u$ as $\hat{u}(x, t)=u(x, t)$. Without this amendment, the microscopic and macroscopic strain profiles as depicted in Figure 6 are obtained, whereby the two strain fields are plotted along the bar for successive time instants. It can be seen that the microscopic strain (depicted on the left of Figure 6) attains realistic values at the boundary where the force is applied. In contrast, the macroscopic strain (depicted on the right) unrealistically tends to zero at the left end of the bar. If the amendment $\hat{u}(x, t)=u(x, t)$ is adopted, the results of Figure 7 are obtained. It can be seen that for this case, the value of the macroscopic strain at the left end is realistic. This demonstrates the importance of using zero higher-order stress on the boundaries in special model 3. Interestingly, the microscopic strains are affected as well: compared to Figure 6, the microscopic strains in Figure 7 are much smoother.

Next, special model 4 is considered. For every time step, two sets of equations must be solved consecutively. The first set of equations are those of classical elasticity, and for the second set two options exist, either in terms of displacements as in Equation (19) or in terms of strains as in Equation (64). Figures 8 and 9 show the strain profiles along the bar for successive time instants. The results of Equation (18) are also shown — they do not differ in the two Figures. Yet again, the importance of using zero higher-order stresses on the boundaries is evident. In Figure 8 the strains following from Equation (19), and using $\partial u / \partial x=0$ as a boundary condition, attain unrealistic 

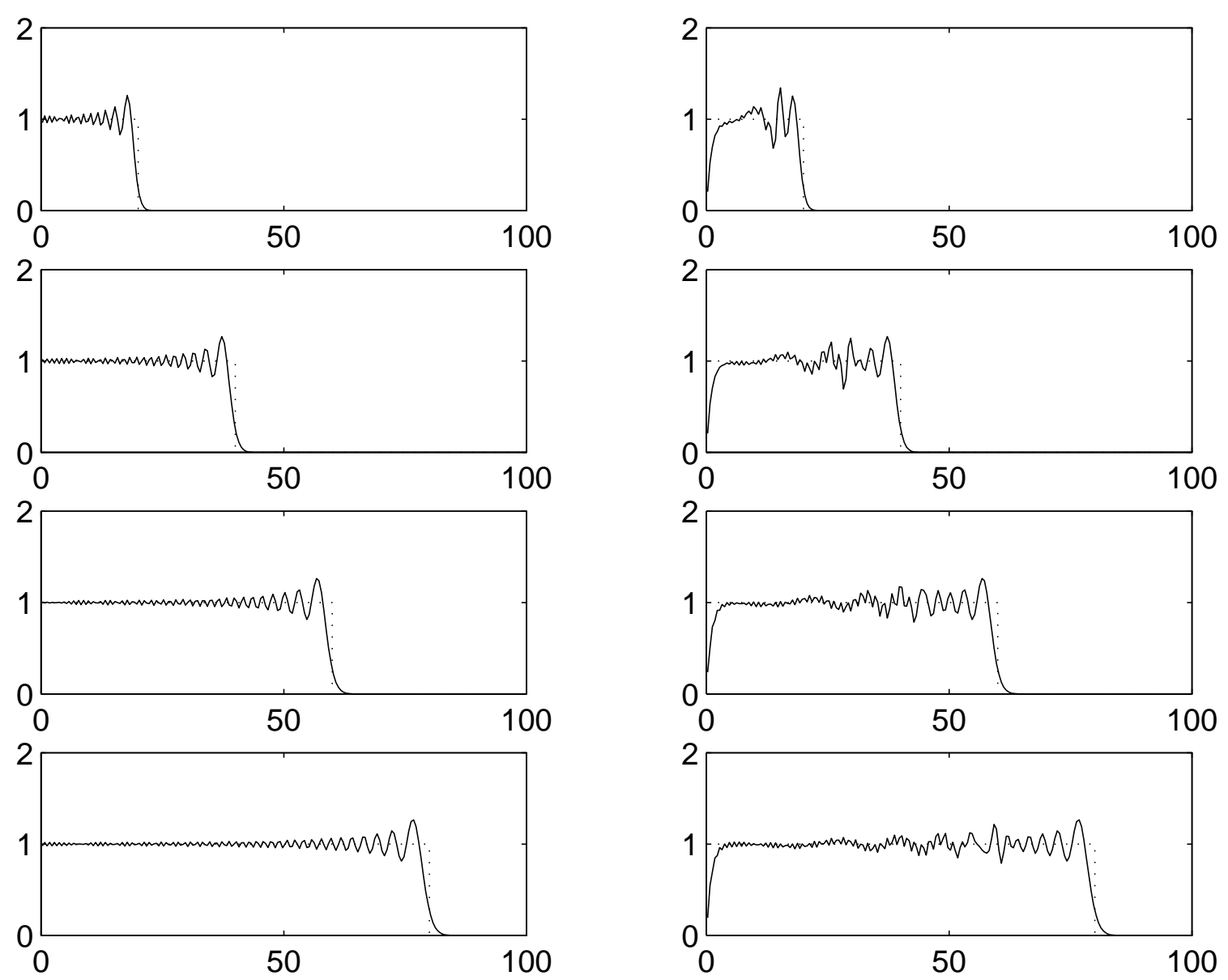

Figure 8: Wave propagation simulated with model 4 - strains for classical elasticity (left) and gradient elasticity (right) across bar for times $20 \mathrm{~s}, 40 \mathrm{~s}, 60 \mathrm{~s}$ and $80 \mathrm{~s}$. Boundary conditions have not been corrected. Dotted lines indicate the analytical solution for classical elasticity.

values at the left end of the bar. In contrast, the strains according to Equation (64), and obtained with the boundary condition $\partial \varepsilon / \partial x=0$, are realistic as is demonstrated in Figure 9.

\subsection{Parameter restriction in model 4}

In Section 4 the dispersive properties of the various special models were presented. For model 4 , the existence of two branches prohibits certain combinations of parameters. In particular, ambiguities arise if the two branches cross. This may happen if $\ell_{1}>\ell_{3}$, in which case the ambiguous wave number $k=1 / \sqrt{\ell_{1}^{2}-\ell_{3}^{2}}$. The corresponding wave length $\lambda$ is found as

$$
\lambda=\frac{2 \pi}{k}=2 \pi \sqrt{\ell_{1}^{2}-\ell_{3}^{2}}
$$

Figure 10 shows the strain profile evolution for an admissible set of parameters, that is $\ell_{1}=1 \mathrm{~m}$ and $\ell_{3}=2 \mathrm{~m}$. Compared to the case $\ell_{1}=\ell_{3}$ (such as the case depicted in Figure 9), the strain profiles for $\ell_{3}>\ell_{1}$ are extremely smooth. However, the situation changes dramatically for an inadmissible set of parameters. In Figure 11 the results are shown for $\ell_{1}=2 \mathrm{~m}$ and $\ell_{3}=1 \mathrm{~m}$. The response is dominated by a single harmonic, of which the amplitude is twice the amplitude of the input signal and the wavelength is given by Equation $(67)$ - in this case, $\lambda \approx 11 \mathrm{~m}$ which is in excellent correspondence with the wave length of the signal observed in Figure 11 (right). This phenomenon is sometimes denoted "internal resonance" and it is emphasized that its occurrence here is completely unphysical. 

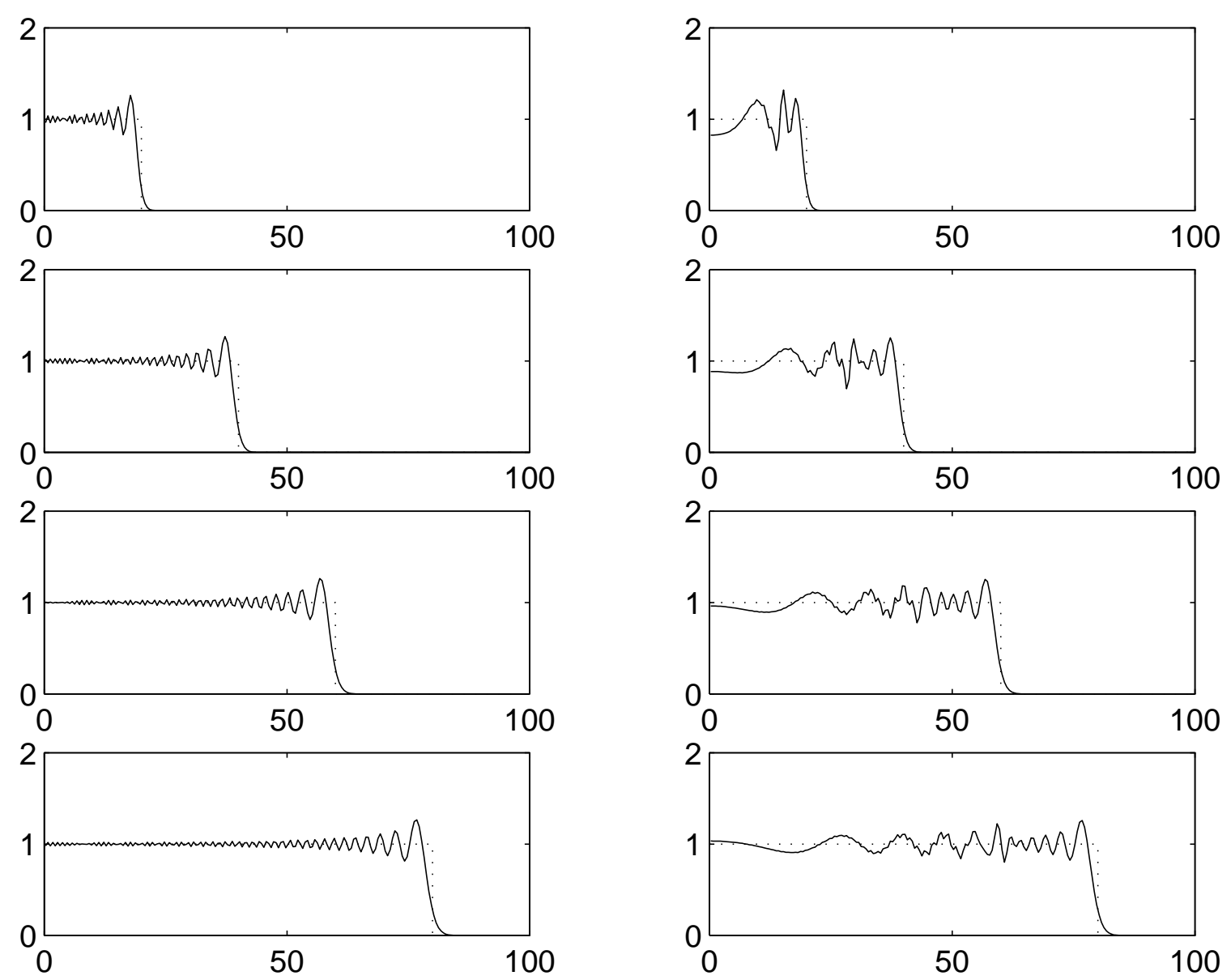

Figure 9: Wave propagation simulated with model 4 - strains for classical elasticity (left) and gradient elasticity (right) across bar for times $20 \mathrm{~s}, 40 \mathrm{~s}, 60 \mathrm{~s}$ and $80 \mathrm{~s}$. Boundary conditions have been corrected by using Equation (64). Dotted lines indicate the analytical solution for classical elasticity.

\subsection{Comparison of special models 1-4}

Finally, the four special models will be compared against one another. The parameter choice of Figure 2 is adopted here, that is $\ell_{1}=\ell_{3}=1 \mathrm{~m}$ and $\ell_{2}=\sqrt{2} \mathrm{~m}$. The same bar problem as above is taken, and in Figure 12 the strain profiles are depicted for all four models and for time instants $t=40 \mathrm{~s}$ and $t=80 \mathrm{~s}$. The most important observation is the qualitative differences between models 1-3 on the one hand and model 4 on the other hand. The higher wave numbers are travelling faster in model 4 than in the other models — in fact, they are travelling with virtually the same velocity as the lower frequencies. Thus, the dispersive properties of model 4 are much less pronounced than those of models 1-3, as already seen in Section 4. The other three models, special models 1-3, differ mainly quantitatively from each other. In all these three models, the lower wave numbers travel faster than the higher wave numbers. The flattening of the wave front is most visible in special model 3.

\section{Concluding remarks}

We have presented four simplified gradient elasticity models that can be used to simulate dispersive wave propagation. The four models are obtained through specific values of the various internal length scales of a generic gradient elasticity formulation that contains three higher-order terms: a fourth-order spatial derivative, a fourthorder time derivative and a mixed fourth-order derivative. The special models are formulated such that they can be implemented using standard finite element procedures. 

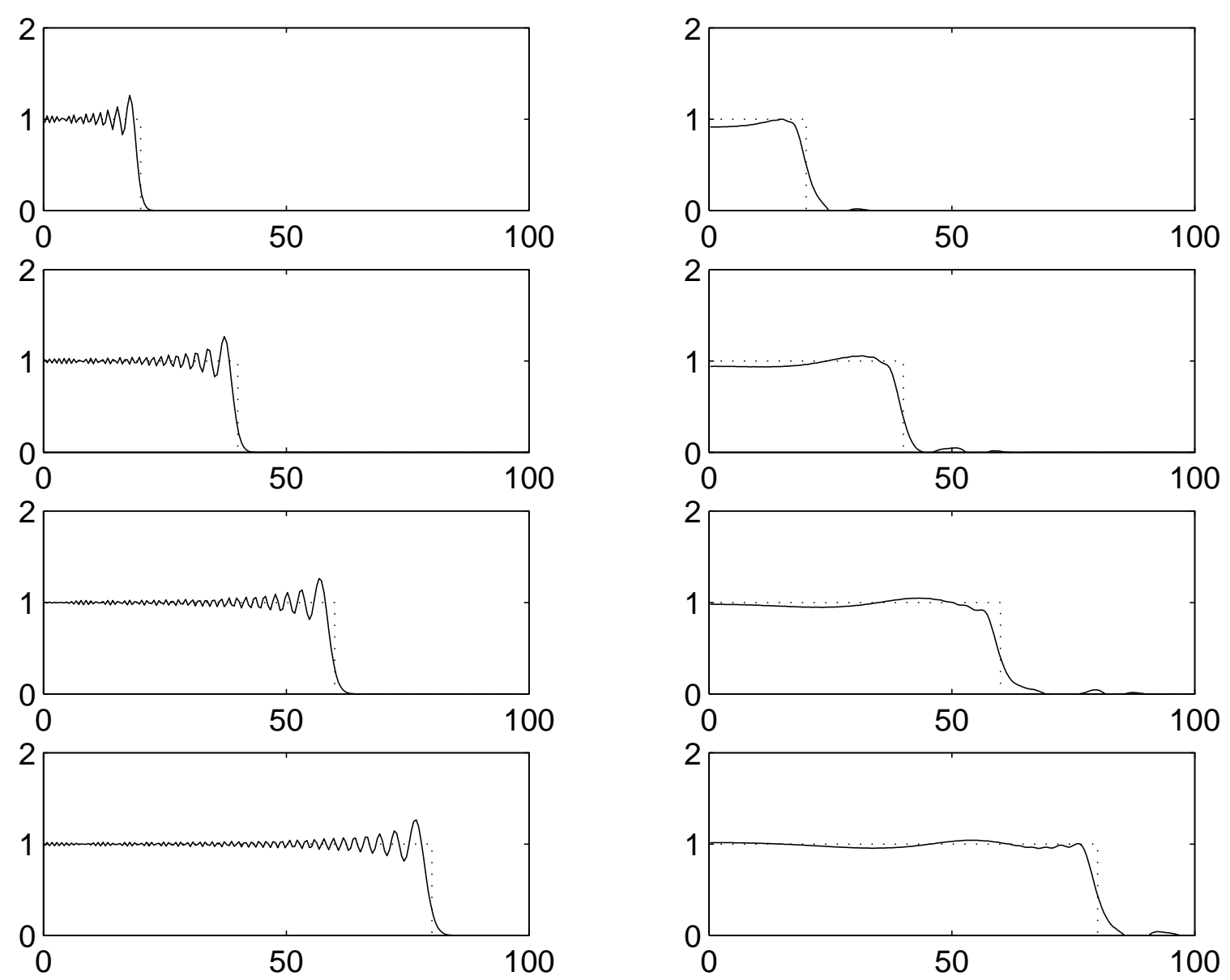

Figure 10: Wave propagation simulated with model 4 - strains for classical elasticity (left) and gradient elasticity (right) across bar for times $20 \mathrm{~s}, 40 \mathrm{~s}, 60 \mathrm{~s}$ and $80 \mathrm{~s}$ with $\ell_{1}=1 \mathrm{~m}$ and $\ell_{3}=2 \mathrm{~m}$.

The four models have been compared in terms of their dispersive behaviour and whether they fulfil Einstein's causality. The dispersive properties of special model 4 are limited in that only the secondary (optical) branch of the curve frequency versus wave number is dispersive; the primary (acoustical) branch is non-dispersive. The other three models exhibit realistic dispersion behaviour. Special models 2 and 4 are strictly causal whereas special models 1 and 3 are not. The causality of the various models is related to the existence of a secondary dispersion curve; if this secondary, optical branch exists and positive real frequencies are obtained for the limit of infinitely large wave numbers, then the model is causal. The variationally consistent boundary conditions have also been presented. For the two special models that are based on a fourth-order spatial derivative (special models 3 and 4), particular attention has been paid to the higher-order boundary conditions. For both models, straightforward and simple amendments have been suggested so as to emulate the effects of zero higher-order stress on the boundary. The importance of such boundary conditions has been demonstrated for both models - failure to impose the correct boundary conditions leads to remarkably similar unrealistic results in the two models.

A final verdict depends on which property is deemed most important. Special model 1 is the simplest from the points of view of implementation and formulation of boundary conditions. Special model 2 is to be preferred if causality is a critical issue. Compared to these two models, special model 3 offers the attractive feature that the propagation velocity of the higher frequency components can be controlled. Although special model 4 retains all three length scales that are present in the original formulation, its dispersive properties are minimal compared to the other three models. 

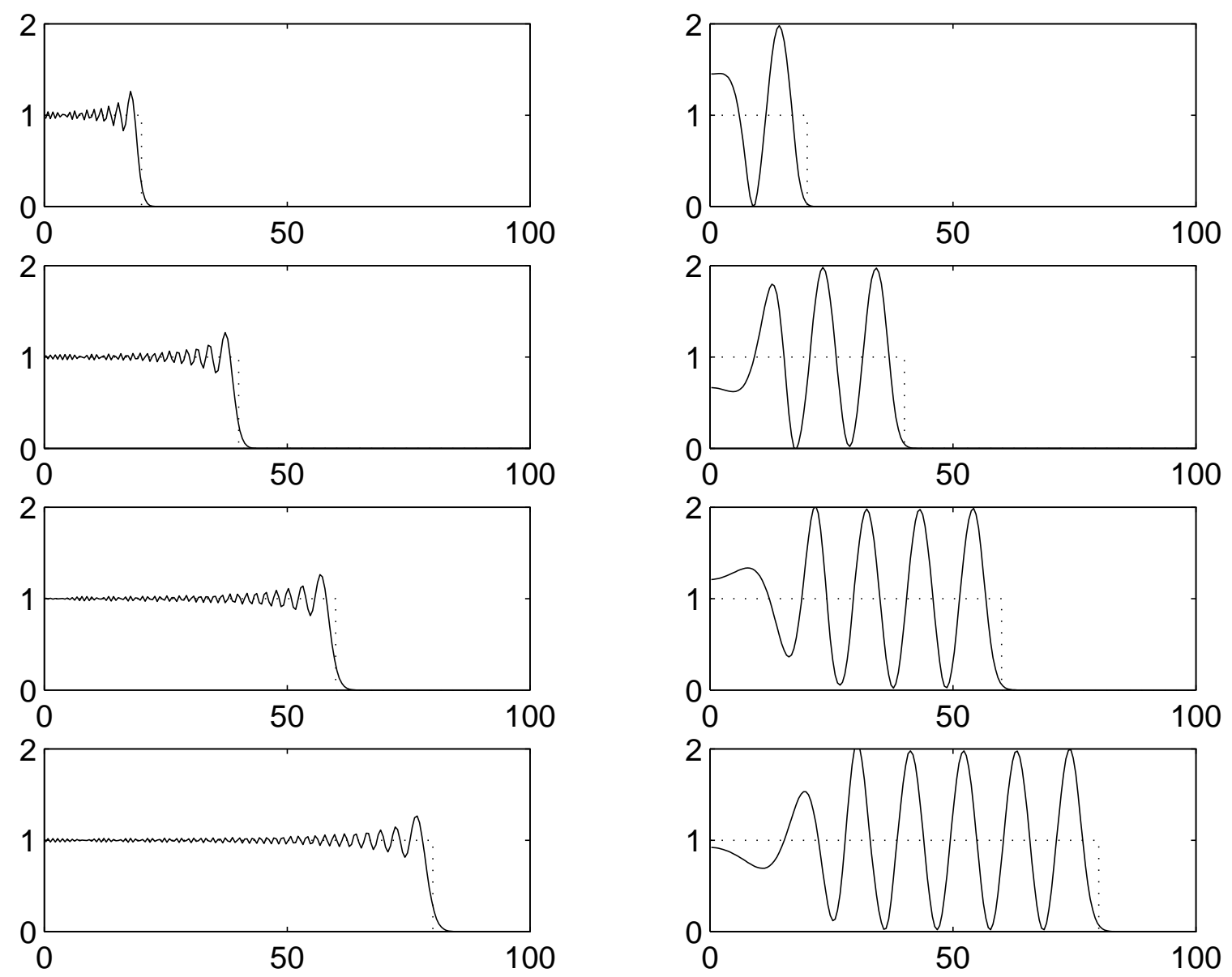

Figure 11: Wave propagation simulated with model 4 - strains for classical elasticity (left) and gradient elasticity (right) across bar for times $20 \mathrm{~s}, 40 \mathrm{~s}, 60 \mathrm{~s}$ and $80 \mathrm{~s}$ with $\ell_{1}=2 \mathrm{~m}$ and $\ell_{3}=1 \mathrm{~m}$.

\section{Acknowledgements}

Financial support of the Engineering and Physical Sciences Research Council to the first author and the fourth author (contract number EP/D041368/1) is gratefully acknowleged.

\section{A Spatial discretisation aspects}

All special models have been rewritten as partial differential equations that are second-order in space, hence spatial discretisation is straightforward. In this section, the spatially discretised systems of equations are treated briefly.

\section{A.1 Special model 1}

The spatial discretisation of special model 1 has been treated in [49] and results in

$$
\left[\mathbf{M}_{0}+\ell_{2}^{2} \mathbf{M}_{1}\right] \frac{\partial^{2} \mathbf{u}}{\partial t^{2}}+\mathbf{K u}=\mathbf{f}
$$

in which $\mathbf{u}$ and $\mathbf{f}$ contain the nodal displacements and the externally applied nodal forces, respectively. Moreover, two mass matrices are defined as

$$
\mathbf{M}_{0}=\int_{0}^{L} \mathbf{N}^{T} \rho \mathbf{N d} x
$$



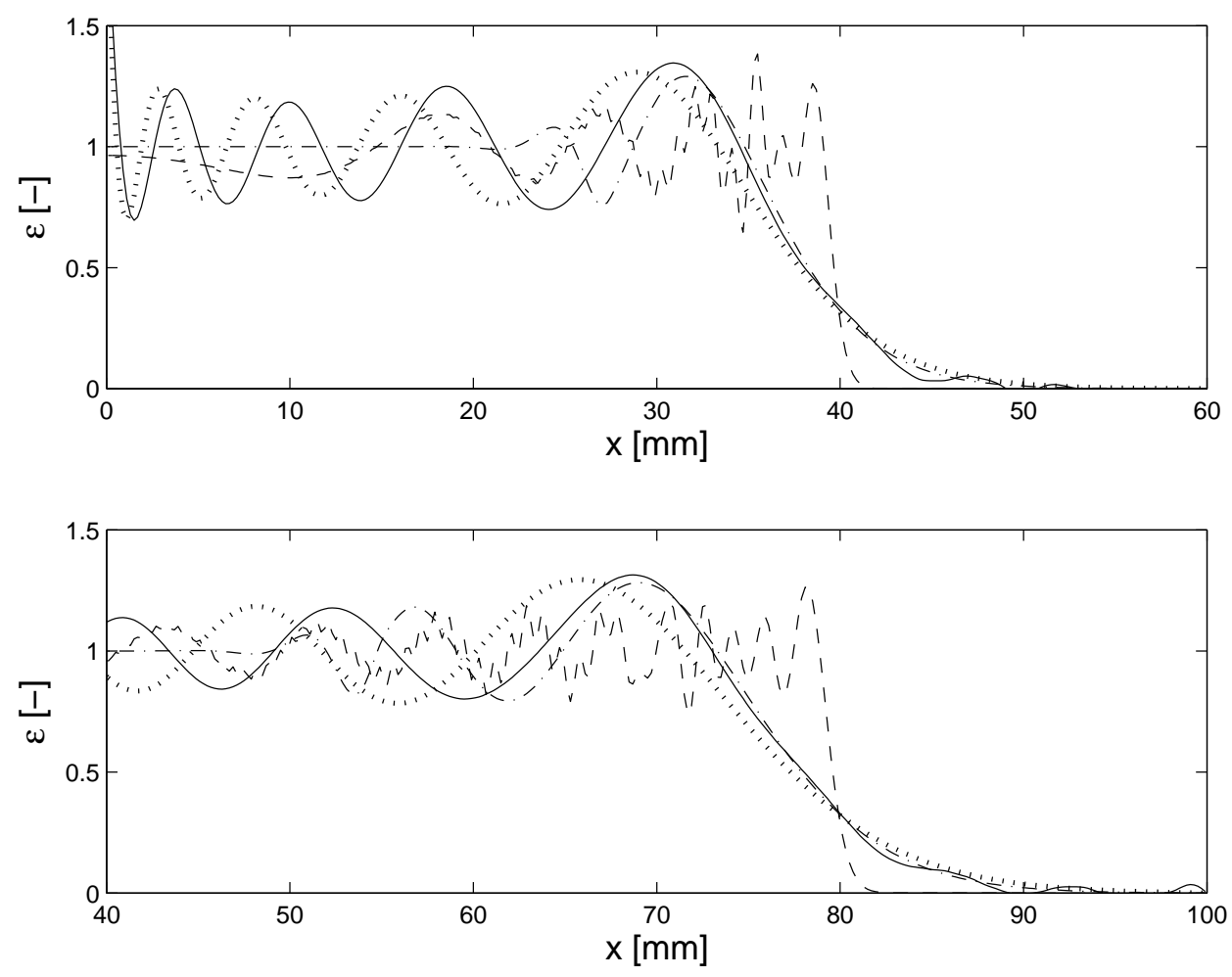

Figure 12: Wave propagation simulated with model 1 (dotted), model 2 (solid), model 3 (dash-dotted) and model 4 (dashed) at time instants $40 \mathrm{~s}$ (top) and $80 \mathrm{~s}$ (bottom)

$$
\mathbf{M}_{1}=\int_{0}^{L} \frac{\partial \mathbf{N}^{T}}{\partial x} \rho \frac{\partial \mathbf{N}}{\partial x} \mathrm{~d} x
$$

and the stiffness matrix is given through

$$
\mathbf{K}=\int_{0}^{L} \frac{\partial \mathbf{N}^{T}}{\partial x} E \frac{\partial \mathbf{N}}{\partial x} \mathrm{~d} x
$$

\section{A.2 Special model 2}

The spatially discretised version of special model 2 is a straightforward extension of Equation (68), i.e.

$$
\left[\mathbf{M}_{0}+\ell_{2}^{2} \mathbf{M}_{1}\right] \frac{\partial^{2} \mathbf{u}}{\partial t^{2}}+\frac{\ell_{1}^{2}}{c_{e}^{2}} \mathbf{M}_{0} \frac{\partial^{4} \mathbf{u}}{\partial t^{4}}+\mathbf{K u}=\mathbf{f}
$$

\section{A.3 Special model 3}

The spatial discretisation of special model 3 has been treated in detail in [31]; in its final version it reads

$$
\left[\begin{array}{cc}
\frac{\ell_{2}^{2}}{\ell_{3}^{2}} & -\frac{\ell_{2}^{2}-\ell_{3}^{2}}{\ell_{3}^{2}} \mathbf{M}_{0} \\
-\frac{\ell_{2}^{2}-\ell_{3}^{2}}{\ell_{3}^{2}} \mathbf{M}_{0}^{T} & \frac{\ell_{2}^{2}-\ell_{3}^{2}}{\ell_{3}^{2}} \mathbf{M}_{0}+\left(\ell_{2}^{2}-\ell_{3}^{2}\right) \mathbf{M}_{1}
\end{array}\right]\left[\begin{array}{l}
\frac{\partial^{2} \hat{\mathbf{u}}}{\partial t^{2}} \\
\frac{\partial^{2} \mathbf{u}}{\partial t^{2}}
\end{array}\right]+\left[\begin{array}{cc}
\mathbf{K} & 0 \\
0 & 0
\end{array}\right]\left[\begin{array}{l}
\hat{\mathbf{u}} \\
\mathbf{u}
\end{array}\right]=\left[\begin{array}{l}
\mathbf{f} \\
0
\end{array}\right]
$$

where it has been assumed that the same shape functions are used for the interpolation of $\hat{u}$ and $u$. 


\section{A.4 Special model 4}

Special model 4 consists of two steps. Firstly, the discretised nodal displacements of classical elasticity $\mathbf{u}_{c}$ are obtained from

$$
\mathbf{M}_{0} \frac{\partial^{2} \mathbf{u}_{c}}{\partial t^{2}}+\mathbf{K} \mathbf{u}_{c}=\mathbf{f}
$$

Afterwards, this solution is used as input in the second equation by which the gradient-dependent nodal displacements $\mathbf{u}$ are found:

$$
\left[\mathbf{H}_{0}+\ell_{3}^{2} \mathbf{H}_{1}\right] \mathbf{u}+\frac{\ell_{1}^{2}}{c_{e}^{2}} \mathbf{H}_{0} \frac{\partial^{2} \mathbf{u}}{\partial t^{2}}=\mathbf{H}_{0} \mathbf{u}_{c}
$$

where

$$
\begin{aligned}
\mathbf{H}_{0} & =\int_{0}^{L} \mathbf{N}^{T} \mathbf{N} \mathrm{d} x \\
\mathbf{H}_{1} & =\int_{0}^{L} \frac{\partial \mathbf{N}^{T}}{\partial x} \frac{\partial \mathbf{N}}{\partial x} \mathrm{~d} x
\end{aligned}
$$

In case the second step of the model is expressed in terms of strains rather than displacements, cf. Equation (64), the right-hand-side of Equation (75) is replaced by $\int \mathbf{N}^{T} \frac{\partial \mathbf{N}}{\partial x} \mathrm{~d} x \mathbf{u}_{c}$.

\section{B Numerical time integration of special model 2}

For the numerical time integration of Equation (11) an extension of the average acceleration variant of the Newmark scheme is developed. It is assumed that, within the time interval $[t, t+\Delta t]$ an average value of the nodal fourth-order time derivatives $\mathbf{c}$ can be defined as

$$
\mathbf{c}_{\mathrm{av}} \equiv \frac{1}{2}\left(\mathbf{c}_{t}+\mathbf{c}_{t+\Delta t}\right)
$$

This quantity is then used in successive integration to obtain the nodal third-order time derivatives $\mathbf{b}$, the nodal accelerations $\mathbf{a}$, the nodal velocities $\mathbf{v}$ and, ultimately, the nodal displacements $\mathbf{u}$ as

$$
\begin{aligned}
\mathbf{b}_{t+\Delta t} & =\mathbf{b}_{t}+\mathbf{c}_{\mathrm{av}} \Delta t \\
\mathbf{a}_{t+\Delta t} & =\mathbf{a}_{t}+\mathbf{b}_{t} \Delta t+\frac{1}{2} \mathbf{c}_{\mathrm{av}} \Delta t^{2} \\
\mathbf{v}_{t+\Delta t} & =\mathbf{v}_{t}+\mathbf{a}_{t} \Delta t+\frac{1}{2} \mathbf{b}_{t} \Delta t^{2}+\frac{1}{6} \mathbf{c}_{\mathrm{av}} \Delta t^{3} \\
\mathbf{u}_{t+\Delta t} & =\mathbf{u}_{t}+\mathbf{v}_{t} \Delta t+\frac{1}{2} \mathbf{a}_{t} \Delta t^{2}+\frac{1}{6} \mathbf{b}_{t} \Delta t^{3}+\frac{1}{24} \mathbf{c}_{\mathrm{av}} \Delta t^{4}
\end{aligned}
$$

\section{References}

[1] Y.L. Yarnell, J.L. Warren, R.G. Wenzel, and S.H. Koenig. Phonon dispersion curves in bismuth. IBM J. Res. Dev., 8:234-240, 1964.

[2] R.D. Mindlin. Micro-structure in linear elasticity. Arch. Rat. Mech. Analysis, 16:52-78, 1964.

[3] R.A. Toupin. Theories for elasticity with couple-stress. Arch. Rat. Mech. Analysis, 17:85-112, 1964.

[4] E.C. Aifantis. On the role of gradients in the localization of deformation and fracture. Int. J. Engng. Sci., 30:1279-1299, 1992. 
[5] S.B. Altan and E.C. Aifantis. On the structure of the mode III crack-tip in gradient elasticity. Scripta Metall. Mater., 26:319-324, 1992.

[6] C.Q. Ru and E.C. Aifantis. A simple approach to solve boundary-value problems in gradient elasticity. Acta Mech., 101:59-68, 1993.

[7] D.J. Unger and E.C. Aifantis. The asymptotic solution of gradient elasticity for mode-III. Int. J. Fract., 71:R27-R32, 1995.

[8] B.S. Altan and E.C. Aifantis. On some aspects in the special theory of gradient elasticity. J. Mech. Behav. Mat., 8:231-282, 1997.

[9] M.Y. Gutkin and E.C. Aifantis. Screw dislocation in gradient elasticity. Scripta Mater., 35:1353-1358, 1996.

[10] M.Y. Gutkin and E.C. Aifantis. Edge dislocation in gradient elasticity. Scripta Mater., 36:129-135, 1997.

[11] M.Y. Gutkin and E.C. Aifantis. Dislocations in the theory of gradient elasticity. Scripta Mater., 40:559-566, 1999.

[12] C.S. Chang and J. Gao. Wave propagation in granular rod using high-gradient theory. ASCE J. Engng. Mech., 123:52-59, 1997.

[13] H.-B. Mühlhaus and F. Oka. Dispersion and wave propagation in discrete and continuous models for granular materials. Int. J. Solids Struct., 33:2841-2858, 1996.

[14] A.S.J. Suiker, de Borst R., and Chang C.S. Micro-mechanical modelling of granular material. Part 1: Derivation of a second-gradient micro-polar constitutive theory. Acta Mech., 149:161-180, 2001.

[15] H. Askes and A.V. Metrikine. Higher-order continua derived from discrete media: continualisation aspects and boundary conditions. Int. J. Solids Struct., 42:187-202, 2005.

[16] H. Askes, A.S.J. Suiker, and L.J. Sluys. A classification of higher-order strain gradient models - linear analysis. Arch. Appl. Mech., 72:171-188, 2002.

[17] J.S. Yang and S.H. Guo. On using strain gradient theories in the analysis of cracks. Int. J. Fract., 133:L19L22, 2005.

[18] H. Askes and E.C. Aifantis. Gradient elasticity theories in statics and dynamics - a unification of approaches. Int. J. Fract., 139:297-304, 2006.

[19] H.G. Georgiadis, I. Vardoulakis, and G. Lykotrafitis. Torsional surface waves in a gradient-elastic half-space. Wave Mot., 31:333-348, 2000.

[20] A.V. Metrikine and H. Askes. One-dimensional dynamically consistent gradient elasticity models derived from a discrete microstructure. Part 1: Generic formulation. Eur. J. Mech. A/Solids, 21:555-572, 2002.

[21] H. Askes and A.V. Metrikine. One-dimensional dynamically consistent gradient elasticity models derived from a discrete microstructure. Part 2: Static and dynamic response. Eur. J. Mech. A/Solids, 21:573-588, 2002.

[22] H.G. Georgiadis. The mode III crack problem in microstructured solids governed by dipolar gradient elasticity: static and dynamic analysis. ASME J. Appl. Mech., 70:517-530, 2003.

[23] I.M. Gitman, H. Askes, and E.C. Aifantis. The representative volume size in static and dynamic micro-macro transitions. Int. J. Fract., 135:L3-L9, 2005.

[24] A.V. Metrikine and H. Askes. An isotropic dynamically consistent gradient elasticity model derived from a 2D lattice. Phil. Mag., 86:3259-3286, 2006.

[25] A.V. Metrikine. On causality of the gradient elasticity models. J. Sound Vibr., 297:727-742, 2006. 
[26] A.V. Pichugin, H. Askes, and A. Tyas. Asymptotic equivalence of homogenisation procedures and fine-tuning of continuum theories. J. Sound Vibr., 313:858-874, 2008.

[27] A. Zervos, P. Papanastasiou, and I. Vardoulakis. A finite element displacement formulation for gradient elastoplasticity. Int. J. Numer. Meth. Engng., 50:1369-1388, 2001.

[28] H. Askes and E.C. Aifantis. Numerical modeling of size effect with gradient elasticity - formulation, meshless discretization and examples. Int. J. Fract., 117:347-358, 2002.

[29] Z. Tang, S. Shen, and S.N. Atluri. Analysis of materials with strain-gradient effects: A Meshless Local Petrov-Galerkin(MLPG) approach, with nodal displacements only. Comp. Mod. Engng. Sci., 4:177-196, 2003.

[30] L.T. Tenek and E.C. Aifantis. A two-dimensional finite element implementation of a special form of gradient elasticity. Comp. Model. Engng. Sci., 3:731-741, 2002.

[31] H. Askes, T. Bennett, and E.C. Aifantis. A new formulation and $C^{0}$-implementation of dynamically consistent gradient elasticity. Int. J. Numer. Meth. Engng., 72:111-126, 2007.

[32] H. Askes and M.A. Gutiérrez. Implicit gradient elasticity. Int. J. Numer. Meth. Engng., 67:400-416, 2006.

[33] A. Zervos. Finite elements for elasticity with microstructure and gradient elasticity. Int. J. Numer. Meth. Engng., 72:564-595, 2008.

[34] A.S.J. Suiker, A.V. Metrikine, and R. de Borst. Comparison of wave propagation characteristics of the Cosserat continuum and corresponding lattice models. Int. J. Solids Struct., 38:1563-1583, 2001.

[35] E. Pasternak and H.-B. Muhlhaus. Generalised homogenisation procedures for granular materials. J. Engng. Math., 52:199-229, 2005.

[36] C.S. Chang and J. Gao. Second-gradient constitutive theory for granular material with random packing structure. Int. J. Solids Struct., 32:2279-2293, 1995.

[37] M.B. Rubin, P. Rosenau, and O. Gottlieb. Continuum model of dispersion caused by an inherent material characteristic length. J. Appl. Phys., 77:4054-4063, 1995.

[38] W. Chen and J. Fish. A dispersive model for wave propagation in periodic heterogeneous media based on homogenization with multiple spatial and temporal scales. ASME J. Appl. Mech., 68:153-161, 2001.

[39] Z.-P. Wang and C.T. Sun. Modeling micro-inertia in heterogeneous materials under dynamic loading. Wave Mot., 36:473-485, 2002.

[40] I.V. Andrianov, J. Awrejcewicz, and R.G. Barantsev. Asymptotic approaches in mechanics: new parameters and procedures. ASME Appl. Mech. Rev., 56:87-110, 2003.

[41] T. Bennett, I.M. Gitman, and H. Askes. Elasticity theories with higher-order gradients of inertia and stiffness for the modelling of wave dispersion in laminates. Int. J. Fract., 148:185-193, 2007.

[42] M. Bunge. Causality; the place of the causal principle in modern science. Harvard University Press, 1959.

[43] J.S. Toll. Causality and the dispersion relation: Logical foundations. Physical Review, 104:1760-1770, 1956.

[44] B.A. Fuchs, B.V. Shabat, and J. Berry J. Functions of complex variables and some of their applications. Pergamon, 1964.

[45] M. Abramowitz and I.A. Stegun. Handbook of mathematical functions; with formulas, graphs, and mathematical tables. Dover, 1970.

[46] J.D. Kaplunov and A.V. Pichugin. On rational boundary conditions for higher-order long-wave models. In F.M. Borodich, editor, IUTAM Symposium on Scaling in Solid Mechanics, pages 81-90. Springer, 2008. ISBN 978-1-4020-9032-5. 
[47] H. Askes, B. Wang, and T. Bennett. Element size and time step selection procedures for the numerical analysis of elasticity with higher-order inertia. J. Sound Vibr., 314:650-656, 2008.

[48] H. Bavinck and H.A. Dieterman. Closed-form dynamic response of damped massspring cascades. J. Comput. Appl. Math., 114:291-303, 2000.

[49] J. Fish, W. Chen, and G. Nagai. Non-local dispersive model for wave propagation in heterogeneous media: one-dimensional case. Int. J. Numer. Meth. Engng., 54:331-346, 2002. 